\title{
Scattering by Magnetic Fields at Large Separation
}

\author{
By \\ Hiroshi T. ITO* and Hideo TAMURA**
}

\section{$\S 1$. Introduction}

In this work we consider the scattering by two magnetic fields with compact support in two dimensions and we analyse the asymptotic behavior of scattering amplitude when the distance between two centers of fields goes to infinity. Even if magnetic fields are of compact support, the magnetic potentials associated with fields do not necessarily fall off rapidly at infinity in the two dimensional space $\boldsymbol{R}^{2}$. This is due to the elementary topological fact that $\boldsymbol{R}^{2} \backslash\{0\}$ is not simply connected. In quantum mechanics, magnetic potentials have a direct significance to the motion of particles as opposed to classical mechanics where the motion is governed only by magnetic fields. This remarkable property is well known as the Aharonov-Bohm effect ([2]). We study how this quantum effect is reflected in the scattering by magnetic fields at large separation. There are many physical literatures on the magnetic scattering in connection to the Aharonov-Bohm effect. We refer to the recent book [1]. A lot of references related to the subject can be found there.

We work in the two dimensional space $\boldsymbol{R}^{2}$ throughout the entire discussion. We denote by $x=\left(x_{1}, x_{2}\right)$ a generic point in $\boldsymbol{R}^{2}$, and we write

$$
H(A)=(-i \nabla-A)^{2}=\sum_{j=1}^{2}\left(-i \partial_{j}-a_{j}\right)^{2}, \quad \partial_{j}=\partial / \partial x_{j},
$$

for the Schrödinger operator with magnetic potential $A(x)=\left(a_{1}(x), a_{2}(x)\right)$ : $\boldsymbol{R}^{2} \rightarrow \boldsymbol{R}^{2}$. The magnetic field $b(x)$ is defined as $b=\nabla \times A=\partial_{1} a_{2}-\partial_{2} a_{1}$

\footnotetext{
Communicated by T. Kawai, November 8, 2000.

2000 Mathematics Subject Classification(s): Primary 81U05; Secondary 35P25, 81Q10.

*Department of Computer Science, Ehime University, Matsuyama 790-8577, Japan.

e-mail: ito@cs.ehime-u.ac.jp

** Department of Mathematics, Okayama University, Okayama 700-8530, Japan.

e-mail: tamura@math.okayama-u.ac.jp
} 
and the quantity $\alpha=(2 \pi)^{-1} \int b(x) d x$ is called the total flux of field $b$, where the integration with no domain attached is taken over the whole space. We often use this abbreviation in the discussion below. For given field $b(x)$, the corresponding potential $A(x)$ is not uniquely determined, but the scattering amplitude is invariant under the gauge transformation $A \rightarrow A+\nabla g$. We fix one of such magnetic potentials. The precise form is specified in Section 2 (Lemma 2.1). As stated at the beginning, magnetic potentials are not in general expected to fall off rapidly at infinity even for fields with compact support. If, for example, we define

$$
A_{b}(x)=\left(a_{1 b}(x), a_{2 b}(x)\right)=\left(-\partial_{2} \varphi(x), \partial_{1} \varphi(x)\right)
$$

with $\varphi=(2 \pi)^{-1} \int \log |x-y| b(y) d y$, then $\nabla \times A_{b}=\Delta \varphi=b$ and $A_{b}$ becomes the potential associated with field $b$. However, if the flux $\alpha$ does not vanish, then $A_{b}(x)$ cannot decay faster than $O\left(|x|^{-1}\right)$. In fact, it behaves like

$$
A_{b}(x)=A_{\alpha}(x)+O\left(|x|^{-2}\right)
$$

at infinity, where $A_{\alpha}=\alpha\left(-x_{2} /|x|^{2}, x_{1} /|x|^{2}\right)$. Thus the difference $H(A)-H_{0}$ between $H(A)$ and the free Hamiltonian $H_{0}=-\Delta$ is a perturbation of longrange class.

We shall formulate the problem more precisely. We are given two magnetic fields $b_{j}, 1 \leq j \leq 2$, with flux $\alpha_{j}$. Assume that $b_{j} \in C_{0}^{\infty}\left(\boldsymbol{R}^{2}\right)$ is a smooth real function with compact support. Let $A_{j}(x), \nabla \times A_{j}=b_{j}$, be the magnetic potential associated with $b_{j}$. We define the Hamiltonian $H_{d}$ as

$$
H_{d}=H\left(A_{1}+A_{2 d}\right)=\left(-i \nabla-A_{1}-A_{2 d}\right)^{2}, \quad A_{2 d}(x)=A_{2}(x-d),
$$

for $d \in \boldsymbol{R}^{2}$ with $|d| \gg 1$, and we denote by $f_{d}(\omega \rightarrow \tilde{\omega} ; E)$ the scattering amplitude for the pair $\left(H_{d}, H_{0}\right)$. The quantity $\left|f_{d}(\omega \rightarrow \tilde{\omega} ; E)\right|^{2}$ is called the differential cross section for scattering from the initial direction $\omega \in S^{1}$ to the final direction $\tilde{\omega}$ at energy $E>0, S^{1}$ being the unit circle. The precise representation for amplitude is given in Section 2 (Lemma 2.4). Our aim is to analyse the asymptotic behavior as $|d| \rightarrow \infty$ of $f_{d}(\omega \rightarrow \tilde{\omega} ; E)$. We denote by $f_{j}(\omega \rightarrow \tilde{\omega} ; E), 1 \leq j \leq 2$, the amplitude for $\left(H\left(A_{j}\right), H_{0}\right)$. Then

$$
f_{2, d}(\omega \rightarrow \tilde{\omega} ; E)=\exp (-i \sqrt{E} d \cdot(\tilde{\omega}-\omega)) f_{2}(\omega \rightarrow \tilde{\omega} ; E)
$$

becomes the amplitude for $\left(H\left(A_{2 d}\right), H_{0}\right)$. We further write $\gamma(x ; \omega)$ for the azimuth angle from direction $\omega \in S^{1}$, and we define $\tau(x ; \omega, \tilde{\omega})$ as

$$
\tau(x ; \omega, \tilde{\omega})=\gamma(x ; \omega)-\gamma(x ;-\tilde{\omega}) .
$$


The main theorem is now stated as follows.

Theorem 1.1. Let the notation be as above. Assume that $\omega \neq \tilde{\omega}$. Fix the direction $\hat{d}=d /|d|$. If $\omega$ and $\tilde{\omega}$ fulfill $\omega \neq \pm \hat{d}$ and $\tilde{\omega} \neq \pm \hat{d}$, then $f_{d}(\omega \rightarrow$ $\tilde{\omega} ; E)$ behaves like

$$
\begin{aligned}
f_{d}(\omega \rightarrow \tilde{\omega} ; E)= & \exp \left(i \alpha_{2} \tau(-d ; \omega, \tilde{\omega})\right) f_{1}(\omega \rightarrow \tilde{\omega} ; E) \\
& +\exp \left(i \alpha_{1} \tau(d ; \omega, \tilde{\omega})\right) f_{2, d}(\omega \rightarrow \tilde{\omega} ; E)+o(1)
\end{aligned}
$$

as $|d| \rightarrow \infty$. In particular, the backward scattering amplitude obeys

$$
f_{d}(\omega \rightarrow-\omega ; E)=f_{1}(\omega \rightarrow-\omega ; E)+f_{2, d}(\omega \rightarrow-\omega ; E)+o(1)
$$

for $\omega \neq \pm \hat{d}$.

As previously stated, the motion of particles in quantum mechanical systems is subject to the influence of magnetic potentials as well as of magnetic fields. This property can be found in the asymptotic formula (1.3). In fact, the phase factor $\exp \left(i \alpha_{1} \tau\left(d ; \omega, \omega^{\prime}\right)\right)$ in front of $f_{2, d}(\omega \rightarrow \tilde{\omega} ; E)$ depends on the flux $\alpha_{1}$ of field $b_{1}$. This means that $b_{1}(x)$ has an influence upon the scattering by field $b_{2 d}(x)=\nabla \times A_{2 d}(x)=b_{2}(x-d)$, although the support of $b_{1}$ is located in the long distance from that of $b_{2 d}$. The magnetic effect is more strongly reflected when $\omega= \pm \hat{d}$ or $\tilde{\omega}= \pm \hat{d}$, and the asymptotic formula is shown to take a slightly different form. We here consider only the special case that at least one of fluxes $\alpha_{1}$ and $\alpha_{2}$ is an integer. The result still holds true for the general case without such a restriction. We are going to study the Aharonov-Bohm effect in the scattering by two $\delta$-like magnetic fields $2 \pi \alpha_{1} \delta(x)$ and $2 \pi \alpha_{2} \delta(x-d)$ with non-integer fluxes $\alpha_{1}$ and $\alpha_{2}$ ([7]). The analysis is based on the idea presented here, although several technical modifications are further required.

We add the new notation to formulate the result. We interpret $\exp (i \alpha \gamma(x ; \omega))$ with $\hat{x}=x /|x|=\omega$ as

$$
\exp (i \alpha \gamma(x ; \omega)):=(1+\exp (i 2 \alpha \pi)) / 2=\cos \alpha \pi \times \exp (i \alpha \pi) .
$$

Then we have the following theorem.

Theorem 1.2. Assume that $\omega \neq \tilde{\omega}$. Fix $\hat{d}=d /|d|$. If at least one of $\alpha_{1}$ and $\alpha_{2}$ is an integer, then (1.3) remains true without the assumption that $\omega \neq \pm \hat{d}$ and $\tilde{\omega} \neq \pm \hat{d}$. In particular, the backward amplitudes satisfy

$$
\begin{aligned}
& f_{d}(\hat{d} \rightarrow-\hat{d} ; E)=f_{1}(\hat{d} \rightarrow-\hat{d} ; E)+\left(\cos \alpha_{1} \pi\right)^{2} f_{2, d}(\hat{d} \rightarrow-\hat{d} ; E)+o(1), \\
& f_{d}(-\hat{d} \rightarrow \hat{d} ; E)=\left(\cos \alpha_{2} \pi\right)^{2} f_{1}(-\hat{d} \rightarrow \hat{d} ; E)+f_{2, d}(-\hat{d} \rightarrow \hat{d} ; E)+o(1) .
\end{aligned}
$$


The present work is motivated by [8] where the same problem has been already studied for the Schrödinger operator $-\Delta+V_{1}(x)+V_{2}(x-d)$ with potentials falling off rapidly at infinity. In the scattering by potentials, the amplitude is completely split into the sum of two amplitudes corresponding to $V_{1}(x)$ and $V_{2}(x-d)$. However the case is quite different in the scattering by magnetic fields in two dimensions. Roughly speaking, the difficulty arises from the long-range property of magnetic potentials. Several new devices are required at many stages in the course of the proof. We introduce various auxiliary operators to approximate $H_{d}$ and the microlocal resolvent estimates for these auxiliary operators play a basic role in proving the theorems. In addition, the proof of Theorem 1.2 makes an essential use of the asymptotic behavior at infinity of the eigenfunction of the Hamiltonian

$$
H_{\alpha}=H\left(A_{\alpha}\right), \quad A_{\alpha}(x)=\alpha\left(-x_{2} /|x|^{2}, x_{1} /|x|^{2}\right),
$$

which has the $\delta$-like magnetic field $\nabla \times A_{\alpha}=2 \pi \alpha \delta(x)$ at the origin. The asymptotic formula of eigenfunction has been already known in the physical literatures $([2],[3],[10])$. The idea seems to extend to the case of several centers. The analysis strongly depends on the location of centers. For example, the extension to the scattering by a finite chain of point-like magnetic fields seems to be interesting. We will discuss the matter in detail elsewhere.

\section{§2. Scattering Amplitudes}

The aim here is to derive the representation for scattering amplitudes in magnetic fields with compact support. The obtained results are stated as Lemmas 2.4 and 2.7. The derivation is based on the idea from [5], [6].

2.1. We begin by constructing a magnetic potential associated with magnetic field $b \in C_{0}^{\infty}\left(\boldsymbol{R}^{2}\right)$ with compact support. For brevity, we assume that $b$ has support in the unit disk $\{|x|<1\}$. Recall that the total flux $\alpha$ is defined by $\alpha=(2 \pi)^{-1} \int b(x) d x$. We shall show that there exists a magnetic potential $A(x) \in C^{\infty}\left(\boldsymbol{R}^{2} \rightarrow \boldsymbol{R}^{2}\right)$ such that

$$
A(x)=A_{\alpha}(x)=\alpha\left(-x_{2} /|x|^{2}, x_{1} /|x|^{2}\right)
$$

for $|x|>2$. To do this, we again set $A_{b}(x)=\left(a_{1 b}(x), a_{2 b}(x)\right)$ as in (1.1). Then $A_{b}(x)$ obeys (1.2). Hence we can define $a_{b}(x)$ as

$$
a_{b}(x)=-\int_{1}^{\infty}\left(x_{1} a_{1 b}(s x)+x_{2} a_{2 b}(s x)\right) d s
$$


for $x \neq 0$. By a simple calculation ([13, Lemma 2.2]), $A_{b}(x)$ is represented as

$$
A_{b}(x)=A_{\alpha}(x)+\nabla a_{b}(x)+E(x)
$$

for $x \neq 0$, where $E(x)=\left(e_{1}(x), e_{2}(x)\right)$ is given by

$$
e_{1}(x)=\int_{1}^{\infty} s x_{2} b(s x) d s, \quad e_{2}(x)=-\int_{1}^{\infty} s x_{1} b(s x) d s
$$

and it vanishes for $|x|>1$. Let $\chi \in C_{0}^{\infty}[0, \infty)$ be a smooth nonnegative cut-off function such that

$$
\chi(s)=1 \quad \text { for } 0 \leq s \leq 1, \quad \chi(s)=0 \text { for } s>2 .
$$

We set $\chi_{0}(x)=\chi(|x|)$ and $\chi_{\infty}(x)=1-\chi_{0}(x)$. Since $E(x)$ vanishes on supp $\chi_{\infty}$, it follows from $(2.2)$ that $A_{b}(x)$ admits the decomposition

$$
A_{b}=\left(\chi_{\infty}+\chi_{0}\right) A_{b}=A(x)+\nabla\left(\chi_{\infty} a_{b}\right),
$$

where

$$
A(x)=\chi_{\infty}(x) A_{\alpha}(x)+B(x)
$$

with $B(x)=a_{b}(x) \nabla \chi_{0}(x)+\chi_{0}(x) A_{b}(x)$. The potential $A(x)$ still has the field $b$ and it satisfies $A(x)=A_{\alpha}(x)$ for $|x|>2$. Thus we have proved the following lemma.

Lemma 2.1. Let $b \in C_{0}^{\infty}\left(\boldsymbol{R}^{2}\right)$ be given smooth magnetic field with total flux $\alpha$. Assume that $b(x)$ has support in $\{|x|<1\}$. Then there exists a smooth magnetic potential $A(x)$ associated with $b$ such that

$$
A(x)=A_{\alpha}(x)=\alpha\left(-x_{2} /|x|^{2}, x_{1} /|x|^{2}\right)
$$

for $|x|>2$.

In the discussion below, we use the notation

$$
\Sigma(R, \omega, \delta)=\{|x|>R, \quad|\hat{x}-\omega|>\delta\}
$$

for $\omega \in S^{1}$, where $\hat{x}=x /|x|$. The azimuth angle $\gamma(x ; \omega)$ from direction $\omega$ satisfies

$$
\nabla \gamma(x ; \omega)=\left(-x_{2} /|x|^{2}, x_{1} /|x|^{2}\right)
$$

and hence it follows from Lemma 2.1 that 


$$
\exp (-i \alpha \gamma(x ; \omega)) H(A) \exp (i \alpha \gamma(x ; \omega))=H(A-\alpha \nabla \gamma)=H_{0}
$$

on $\Sigma(R, \omega, \delta)$, provided that $R \gg 1$. This relation is often used in the future discussion.

2.2. Let $A(x)$ be fixed as in Lemma 2.1. We discuss the scattering theory for the pair $\left(H(A), H_{0}\right)$. The potential $A(x)$ behaves like $A(x)=O\left(|x|^{-1}\right)$ at infinity, so that $H(A)-H_{0}$ is a perturbation of long-range class. Nevertheless we know $([9])$ that the ordinary wave operators

$$
W_{ \pm}\left(H(A), H_{0}\right)=s-\lim _{t \rightarrow \pm \infty} \exp (i t H(A)) \exp \left(-i t H_{0}\right)
$$

exist and are asymptotically complete

$$
\operatorname{Ran} W_{-}\left(H(A), H_{0}\right)=\operatorname{Ran} W_{+}\left(H(A), H_{0}\right) .
$$

Hence the scattering operator

$$
S\left(H(A), H_{0}\right)=W_{+}^{*}\left(H(A), H_{0}\right) W_{-}\left(H(A), H_{0}\right)
$$

can be defined as a unitary operator on $L^{2}\left(\boldsymbol{R}^{2}\right)$.

Let $E>0$ be fixed. We choose $\delta, 0<\delta \ll 1$, sufficiently small and define

$$
\beta_{0}(\xi)=\chi\left(2|\xi-\sqrt{E} \omega| / \delta^{2}\right)
$$

for initial direction $\omega \in S^{1}$, where $\chi \in C_{0}^{\infty}[0, \infty)$ is the cut-off function with property (2.3). We further take a smooth real function $j_{0}(x)$ such that

$$
\operatorname{supp} j_{0} \subset \Sigma(R, \omega, \delta), \quad j_{0}=1 \text { on } \Sigma(2 R, \omega, 2 \delta) .
$$

We may assume that $j_{0}$ obeys $\partial_{x}^{\beta} j_{0}(x)=O\left(|x|^{-|\beta|}\right)$ at infinity. The next lemma is well known ([11]). We skip the proof.

Lemma 2.2. Let $f \in L^{2}\left(\boldsymbol{R}^{2}\right)$. Then the free solution $\exp \left(-i t H_{0}\right) f$ behaves like

$$
\left(\exp \left(-i t H_{0}\right) f\right)(x)=(2 i t)^{-1} \exp \left(i|x|^{2} / 4 t\right) \widehat{f}(x / 2 t)+o(1), \quad|t| \rightarrow \infty,
$$

in $L^{2}\left(\boldsymbol{R}^{2}\right)$, where

$$
\widehat{f}(\xi)=(2 \pi)^{-1} \int e^{-i x \cdot \xi} f(x) d x
$$

denotes the Fourier transform of $f(x)$. 
We introduce the new notation. Let $H_{1}$ and $H_{2}$ be two self-adjoint operators with the same domain in $L^{2}\left(\boldsymbol{R}^{2}\right)$. Then we define $W_{ \pm}\left(H_{2}, H_{1} ; J\right)$ by

$$
W_{ \pm}\left(H_{2}, H_{1} ; J\right)=s-\lim _{t \rightarrow \pm \infty} \exp \left(i t H_{2}\right) J \exp \left(-i t H_{1}\right)
$$

for a bounded operator $J$ on $L^{2}\left(\boldsymbol{R}^{2}\right)$. By Lemma 2.2, it follows that

$$
W_{-}\left(H(A), H_{0}\right) \beta_{0}^{2}=W_{-}\left(H(A), H_{0} ; J\right)
$$

with $J=j_{0}^{2} \beta_{0}^{2}$, where $\beta_{0}=\beta_{0}\left(D_{x}\right)$. Hence we have the decomposition

$$
W_{-}\left(H(A), H_{0}\right) \beta_{0}^{2}=W_{-}\left(H(A), H_{0} ; J_{0}\right) W_{-}\left(H_{0}, H_{0} ; J_{1}\right),
$$

where

$$
J_{0}=j_{0} \exp (i \alpha \gamma(x ; \omega)) \beta_{0}, \quad J_{1}=j_{0} \exp (-i \alpha \gamma(x ; \omega)) \beta_{0} .
$$

The existence of $W_{-}\left(H(A), H_{0} ; J_{0}\right)$ is verified by use of (2.6), while the existence of $W_{-}\left(H_{0}, H_{0} ; J_{1}\right)$ follows from Lemma 2.2. We note that $W_{+}\left(H(A), H_{0} ; J_{0}\right)=$ 0 , which also follows from Lemma 2.2. The same argument applies to final direction $\tilde{\omega} \in S^{1}$. We define

$$
\tilde{\beta}_{0}(\xi)=\chi\left(2|\xi-\sqrt{E} \tilde{\omega}| / \delta^{2}\right)
$$

and we take a real function $\tilde{j}_{0}(x)$ such that

$$
\operatorname{supp} \tilde{j}_{0} \subset \Sigma(R,-\tilde{\omega}, \delta), \quad \tilde{j}_{0}=1 \text { on } \Sigma(2 R,-\tilde{\omega}, 2 \delta) .
$$

If we set

$$
\tilde{J}_{0}=\tilde{j}_{0} \exp (i \alpha \gamma(x ;-\tilde{\omega})) \tilde{\beta}_{0}, \quad \tilde{J}_{1}=\tilde{j}_{0} \exp (-i \alpha \gamma(x ;-\tilde{\omega})) \tilde{\beta}_{0},
$$

then we obtain

$$
W_{+}\left(H(A), H_{0}\right) \tilde{\beta}_{0}^{2}=W_{+}\left(H(A), H_{0} ; \tilde{J}_{0}\right) W_{+}\left(H_{0}, H_{0} ; \tilde{J}_{1}\right) .
$$

2.3. We proceed to the representation of scattering amplitude for $\left(H(A), H_{0}\right)$. The operator $H(A)$ is known to have the following spectral properties ([4]): (1) $H(A)$ has no positive bound state energies; (2) The resolvent

$$
R(\lambda \pm i \varepsilon ; H(A))=(H(A)-\lambda \mp i \varepsilon)^{-1}, \quad \varepsilon>0,
$$

has the boundary values

$$
R(\lambda \pm i 0 ; H(A))=\lim _{\varepsilon \rightarrow 0} R(\lambda \pm i \varepsilon ; H(A)), \quad \lambda>0,
$$


to the positive axis as an operator from the space $L_{s}^{2}\left(\boldsymbol{R}^{2}\right)=L^{2}\left(\boldsymbol{R}^{2} ;\langle x\rangle^{2 s} d x\right)$ into $L_{-s}^{2}\left(\boldsymbol{R}^{2}\right)$ for $s>1 / 2$, where $\langle x\rangle=\left(1+|x|^{2}\right)^{1 / 2}$. The second property is known as the principle of limiting absorption. Let

$$
\varphi_{0}(x ; \theta, \lambda)=\exp (i \sqrt{\lambda} x \cdot \theta), \quad \lambda>0, \quad \theta \in S^{1},
$$

be the generalized eigenfunction of $H_{0}, H_{0} \varphi_{0}=\lambda \varphi_{0}$. If we define the unitary mapping $F: L^{2}\left(\boldsymbol{R}^{2}\right) \rightarrow L^{2}((0, \infty) ; d \lambda) \otimes L^{2}\left(S^{1}\right)$ by

$$
(F u)(\lambda, \theta)=2^{-1 / 2}(2 \pi)^{-1} \int \bar{\varphi}_{0}(x ; \theta, \lambda) u(x) d x=2^{-1 / 2} \hat{u}(\sqrt{\lambda} \theta),
$$

then $H_{0}$ is diagonalized as $F H_{0} F^{*}=\lambda \times$ on $L^{2}((0, \infty) ; d \lambda) \otimes L^{2}\left(S^{1}\right)$ and the scattering operator $S\left(H(A), H_{0}\right)$ is decomposed into the direct integral

$$
S\left(H(A), H_{0}\right) \simeq F S\left(H(A), H_{0}\right) F^{*}=\int_{0}^{\infty} \oplus S\left(\lambda ; H(A), H_{0}\right) d \lambda,
$$

where the fibre $S\left(\lambda ; H(A), H_{0}\right): L^{2}\left(S^{1}\right) \rightarrow L^{2}\left(S^{1}\right)$ is called the scattering matrix at energy $\lambda>0$ and it acts as

$$
\left(F S\left(H(A), H_{0}\right) u\right)(\lambda, \theta)=\left(S\left(\lambda ; H(A), H_{0}\right)(F u)(\lambda, \cdot)\right)(\theta)
$$

for $u \in L^{2}\left(\boldsymbol{R}^{2}\right)$.

We combine (2.8) and (2.10) to obtain that

$$
\tilde{\beta}_{0}^{2} S\left(H(A), H_{0}\right) \beta_{0}^{2}=W_{+}^{*}\left(H_{0}, H_{0} ; \tilde{J}_{1}\right) S_{0}\left(H(A), H_{0}\right) W_{-}\left(H_{0}, H_{0} ; J_{1}\right),
$$

where

$$
S_{0}\left(H(A), H_{0}\right)=W_{+}^{*}\left(H(A), H_{0} ; \tilde{J}_{0}\right) W_{-}\left(H(A), H_{0} ; J_{0}\right) .
$$

We see from Lemma 2.2 that $W_{-}\left(H_{0}, H_{0} ; J_{1}\right)$ acts as

$$
F W_{-}\left(H_{0}, H_{0} ; J_{1}\right) F^{*}=\exp (-i \alpha \gamma(-\theta ; \omega)) \beta_{0}(\sqrt{\lambda} \theta) \times
$$

on $L^{2}((0, \infty) ; d \lambda) \otimes L^{2}\left(S^{1}\right)$. Similarly we have

$$
F W_{+}\left(H_{0}, H_{0} ; \tilde{J}_{1}\right) F^{*}=\exp (-i \alpha \gamma(\theta ;-\tilde{\omega})) \tilde{\beta}_{0}(\sqrt{\lambda} \theta) \times .
$$

We note that

$$
e^{-i \alpha \gamma(-\omega ; \omega)} \beta_{0}(\sqrt{E} \omega)=e^{-i \alpha \pi}, \quad e^{-i \alpha \gamma(\tilde{\omega} ;-\tilde{\omega})} \tilde{\beta}_{0}(\sqrt{E} \tilde{\omega})=e^{-i \alpha \pi}
$$

The operator $S_{0}\left(H(A), H_{0}\right)$ commutes with $H_{0}$, and hence it also has the direct integral decomposition. We denote by $S\left(\theta^{\prime}, \theta ; \lambda\right)$ the kernel of $S\left(\lambda ; H(A), H_{0}\right)$ 
and by $S_{0}\left(\theta^{\prime}, \theta ; \lambda\right)$ the kernel of fibre $S_{0}\left(\lambda ; H(A), H_{0}\right): L^{2}\left(S^{1}\right) \rightarrow L^{2}\left(S^{1}\right)$ of $S_{0}\left(H(A), H_{0}\right)$. Then it follows from $(2.11)$ and $(2.12)$ that $S(\tilde{\omega}, \omega ; E)=$ $S_{0}(\tilde{\omega}, \omega ; E)$. We shall derive the representation for $S_{0}\left(\theta^{\prime}, \theta ; \lambda\right)$. To do this, we follow the idea due to [6]. We calculate $T=H(A) J_{0}-J_{0} H_{0}$ as

$$
T=\exp (i \alpha \gamma(x ; \omega))\left(H_{0} j_{0}-j_{0} H_{0}\right) \beta_{0}=\exp (i \alpha \gamma(x ; \omega))\left[H_{0}, j_{0}\right] \beta_{0}
$$

by use of (2.6). Similarly we have

$$
\tilde{T}=H(A) \tilde{J}_{0}-\tilde{J}_{0} H_{0}=\exp (i \alpha \gamma(x ;-\tilde{\omega}))\left[H_{0}, \tilde{j}_{0}\right] \tilde{\beta}_{0} .
$$

Since $W_{+}\left(H(A), H_{0} ; J_{0}\right)=0$, we have

$$
W_{-}\left(H(A), H_{0} ; J_{0}\right)=-i \int_{-\infty}^{\infty} \exp (i t H(A)) T \exp \left(-i t H_{0}\right) d t .
$$

If we make use of this relation, the lemma below can be verified in exactly the same way as [6, Theorem 3.3].

Lemma 2.3. Let the notation be as above. Then $S_{0}\left(\lambda ; H(A), H_{0}\right)$ : $L^{2}\left(S^{1}\right) \rightarrow L^{2}\left(S^{1}\right)$ has the representation

$$
S_{0}\left(\lambda ; H(A), H_{0}\right)=2 \pi i \gamma_{0}(\lambda)\left(-\tilde{J}_{0}^{*} T+\tilde{T}^{*} R(\lambda+i 0 ; H(A)) T\right) \gamma_{0}^{*}(\lambda),
$$

where $\gamma_{0}(\lambda)$ is the trace operator defined by

$$
\left(\gamma_{0}(\lambda) f\right)(\theta)=(F f)(\lambda, \theta)=2^{-1 / 2} \widehat{f}(\sqrt{\lambda} \theta): L_{s}^{2}\left(\boldsymbol{R}^{2}\right) \rightarrow L^{2}\left(S^{1}\right), \quad s>1 / 2 .
$$

We note that the relation in the lemma makes sense. By (2.13) and (2.14), $T$ and $\tilde{T}$ are both realized as pseudodifferential operators $T=T\left(x, D_{x}\right)$ and $\tilde{T}=\tilde{T}\left(x, D_{x}\right)$. By $(2.7), \nabla j_{0}$ vanishes on $\Sigma(2 R, \omega, 2 \delta)$, and $\xi \in \operatorname{supp} \beta_{0}$ takes values around $\sqrt{E} \omega$. Hence $(x, \xi) \in \operatorname{supp} T$ has the outgoing property $\hat{x} \cdot \hat{\xi}>1 / 2$ for $|x| \gg 1$. On the other hand, $(x, \xi) \in \operatorname{supp} \tilde{T}$ has the incoming property $\hat{x} \cdot \hat{\xi}<$ $-1 / 2$. Then it follows from the microlocal resolvent estimate [5, Theorems 1 and 2] (see [15, Theorem 9.2] also) that

$$
\langle x\rangle^{N} \tilde{T}^{*} R(\lambda+i 0 ; H(A)) T\langle x\rangle^{N}: L^{2} \rightarrow L^{2}
$$

is bounded for any $N \gg 1$. The symbols $\tilde{J}_{0}(x, \xi)$ and $T(x, \xi)$ have support around $\sqrt{E} \tilde{\omega}$ and $\sqrt{E} \omega$ in the $\xi$ variables respectively. Since $\omega \neq \tilde{\omega}$, we can take $\delta$ so small that the supports of two symbols do not intersect with each other, and hence 


$$
\langle x\rangle^{N} \tilde{J}_{0}^{*} T\langle x\rangle^{N}: L^{2} \rightarrow L^{2}
$$

is also bounded by a simple calculus of pseudodifferential operators. Thus the relation in Lemma 2.3 makes sense.

The scattering amplitude $f(\omega \rightarrow \tilde{\omega} ; E)$ for scattering from initial direction $\omega$ to final one $\tilde{\omega}$ at energy $E>0$ is defined by

$$
f(\omega \rightarrow \tilde{\omega} ; E)=c(E)(S(\tilde{\omega}, \omega ; E)-\delta(\tilde{\omega}-\omega))
$$

with $c(E)=(2 \pi / i \sqrt{E})^{1 / 2}$. We denote by $($,$) the L^{2}$ scalar product. Then the next lemma follows from Lemma 2.3 at once.

Lemma 2.4. Assume that $\omega \neq \tilde{\omega}$. Then $f(\omega \rightarrow \tilde{\omega} ; E)$ is represented as

$$
\begin{aligned}
f(\omega \rightarrow \tilde{\omega} ; E)= & -(i c(E) / 4 \pi)\left(T \varphi_{0}(\omega, E), \tilde{J}_{0} \varphi_{0}(\tilde{\omega}, E)\right) \\
& +(i c(E) / 4 \pi)\left(R(E+i 0 ; H(A)) T \varphi_{0}(\omega, E), \tilde{T} \varphi_{0}(\tilde{\omega}, E)\right),
\end{aligned}
$$

where we write $\varphi_{0}(\omega, E)$ for $\varphi_{0}(x ; \omega, E)=\exp (i \sqrt{E} x \cdot \omega)$.

2.4. We now fix $\sigma, 0<\sigma \ll 1$, small enough and take $R$ as $R=|d|^{\sigma}$, $|d| \gg 1$, in (2.7) and (2.9). Then $j_{0}$ has support on $\Sigma\left(|d|^{\sigma}, \omega, \delta\right)$ and $j_{0}=$ 1 on $\Sigma\left(2|d|^{\sigma}, \omega, 2 \delta\right)$ for $0<\delta \ll 1$ small enough, while $\tilde{j}_{0}$ has support on $\Sigma\left(|d|^{\sigma},-\tilde{\omega}, \delta\right)$ and $\tilde{j}_{0}=1$ on $\Sigma\left(2|d|^{\sigma},-\tilde{\omega}, 2 \delta\right)$. We may assume that $j_{0}(x)$ satisfies $\partial_{x}^{\beta} j_{0}=O\left(|x|^{-|\beta|}\right)$ uniformly in $d$; similarly for $\tilde{j}_{0}$. Since the operator in (2.16) is bounded uniformly in $d$, it follows that

$$
\left(T \varphi_{0}(\omega, E), \tilde{J}_{0} \varphi_{0}(\tilde{\omega}, E)\right)=O\left(|d|^{-N}\right), \quad|d| \rightarrow \infty,
$$

for any $N \gg 1$. Thus we have

$$
f(\omega \rightarrow \tilde{\omega} ; E)=(i c(E) / 4 \pi)\left(R(E+i 0 ; H(A)) T \varphi_{0}(\omega, E), \tilde{T} \varphi_{0}(\tilde{\omega}, E)\right)+o(1)
$$

as $|d| \rightarrow \infty$. The operator in (2.15) is not necessarily bounded uniformly in $d$. We continue to analyse the term on the right side of (2.17). We decompose $T=T\left(x, D_{x}\right)$ into

$$
T=\chi_{1 d} T+\left(1-\chi_{1 d}\right) T=T_{1}+T_{2},
$$

where $\chi_{1 d}(x)=\chi\left(|x| / 3|d|^{\sigma}\right)$. Similarly $\tilde{T}=\tilde{T}_{1}+\tilde{T}_{2}$. Since $\nabla j_{0}$ vanishes on $\Sigma\left(2|d|^{\sigma}, \omega, 2 \delta\right), T_{2}(x, \xi)$ has the support in the set

$$
\operatorname{supp} T_{2} \subset\left\{|x|>3|d|^{\sigma}, \quad|\hat{x}-\omega|<2 \delta, \quad|\xi-\sqrt{E} \omega|<\delta^{2}\right\}
$$


of the phase space $\boldsymbol{R}_{x}^{2} \times \boldsymbol{R}_{\xi}^{2}$, and $(x, \xi) \in \operatorname{supp} T_{2}$ has the outgoing property $\hat{x} \cdot \hat{\xi}>0$. On the other hand, $\tilde{T}_{2}(x, \xi)$ has the support in the set

$$
\operatorname{supp} \tilde{T}_{2} \subset\left\{|x|>3|d|^{\sigma}, \quad|\hat{x}+\tilde{\omega}|<2 \delta, \quad|\xi-\sqrt{E} \tilde{\omega}|<\delta^{2}\right\}
$$

and $(x, \xi) \in \operatorname{supp} \tilde{T}_{2}$ has the incoming property $\hat{x} \cdot \hat{\xi}<0$.

Lemma 2.5. Let $T_{k}, \tilde{T}_{k}, 1 \leq k \leq 2$, be as above. Then

$$
\left\|\tilde{T}_{k}^{*} R(E+i 0 ; H(A)) T_{2}\right\|=O\left(|d|^{-N}\right), \quad\left\|\tilde{T}_{2}^{*} R(E+i 0 ; H(A)) T_{k}\right\|=O\left(|d|^{-N}\right)
$$

for any $N \gg 1$, where \|\| denotes the norm of bounded operators acting on $L^{2}$.

To prove this, we use the following lemma.

Lemma 2.6. $\quad$ Let $r_{L}$ be the multiplication by

$$
r_{L}=r_{L}(x ; d)=\left(|x|^{2}+|d|^{2}\right)^{-L / 2}
$$

with $L \gg 1$. Let $g_{ \pm}(x)$ and $f_{ \pm}(\xi)$ be smooth functions such that $\left|\partial_{x}^{\beta} g_{ \pm}(x)\right| \leq C_{\beta}$ and

$$
\operatorname{supp} g_{ \pm} \subset\{|x|>c\}, \quad \operatorname{supp} f_{ \pm} \subset\{1 / c<|\xi|<c\}
$$

for some $c>1$. Assume that there exists $\mu, 0<\mu<1$, such that $\pm \hat{x} \cdot \hat{\xi}>-\mu$ for $(x, \xi) \in \operatorname{supp} g_{ \pm} \times \operatorname{supp} f_{ \pm}$. If $L>3(s+1)$ for $s>0$, then the resolvent $R\left(E+i 0 ; H_{0}\right)$ of the free Hamiltonian $H_{0}=-\Delta$ obeys

$$
\left\|r_{L} R\left(E+i 0 ; H_{0}\right) f_{+} g_{+}\langle x\rangle^{s}\right\|+\left\|r_{L} R\left(E-i 0 ; H_{0}\right) f_{-} g_{-}\langle x\rangle^{s}\right\|=O\left(|d|^{-2 L / 3}\right),
$$

where $f_{ \pm}=f_{ \pm}\left(D_{x}\right)$.

Proof. The lemma follows from [5, Theorem 1.2]. According to the theorem (see also the proof there), we have that

$$
\langle x\rangle^{-s-\tau} R\left(E \pm i 0 ; H_{0}\right) f_{ \pm} g_{ \pm}\langle x\rangle^{s}: L^{2} \rightarrow L^{2}
$$

is bounded for $s \geq 0$ and $\tau>1$, which completes the proof.

Proof of Lemma 2.5. We construct an approximation for $R(E+i 0 ; H(A))$ $\times T_{2}$. Let $j(x)=j(x ; d)$ be a smooth function such that $\partial_{x}^{\beta} j(x)=O\left(|x|^{-|\beta|}\right)$ uniformly in $d$ and

$$
\operatorname{supp} j \subset \Sigma\left(|d|^{\sigma},-\omega, \delta\right), \quad j=1 \text { on } \Sigma\left(2|d|^{\sigma},-\omega, 2 \delta\right) .
$$


Then $j T_{2}=T_{2}$ and we have the relation

$$
\begin{aligned}
(H(A)-E) j e^{i \alpha \gamma(x ;-\omega)} & =e^{i \alpha \gamma(x ;-\omega)}\left(H_{0}-E\right) j \\
& =e^{i \alpha \gamma(x ;-\omega)}\left(j\left(H_{0}-E\right)+\left[H_{0}, j\right]\right)
\end{aligned}
$$

by use of (2.6). We denote by $\tilde{r}_{L}$ an operator such that

$$
\tilde{r}_{L}\left(|x|^{2}+|d|^{2}\right)^{L / 2}, \quad\left(|x|^{2}+|d|^{2}\right)^{L / 2} \tilde{r}_{L}: L^{2} \rightarrow L^{2}
$$

are both bounded uniformly in $d$. If we set

$$
\beta_{1}(\xi)=\chi\left(|\xi-\sqrt{E} \omega| / \delta^{2}\right),
$$

then $\left(1-\beta_{1}\right) \beta_{0}=0$, and hence it follows that $\left(1-\beta_{1}\right) T_{2}=\tilde{r}_{N}$ for any $N \gg 1$, where $\beta_{1}=\beta_{1}\left(D_{x}\right)$. By $(2.19)$, we have

$$
\begin{aligned}
& (H(A)-E) j e^{i \alpha \gamma(x ;-\omega)} R\left(E+i 0 ; H_{0}\right) \beta_{1} e^{-i \alpha \gamma(x ;-\omega)} T_{2} \\
& \quad=T_{2}+\tilde{r}_{N}+e^{i \alpha \gamma(x ;-\omega)}\left[H_{0}, j\right] R\left(E+i 0 ; H_{0}\right) \beta_{1} e^{-i \alpha \gamma(x ;-\omega)} T_{2} .
\end{aligned}
$$

By definition, $\nabla j$ has support in

$$
\Pi_{+}=\left\{|x| \leq 2|d|^{\sigma}\right\} \cup\left\{|x|>2|d|^{\sigma}, \quad|\hat{x}+\omega|<2 \delta\right\},
$$

while $\left(1-\chi_{1 d}\right) \nabla j_{0}$ has support in

$$
\Pi_{-}=\left\{|x|>3|d|^{\sigma}, \quad|\hat{x}-\omega|<2 \delta\right\} .
$$

We now consider the operator $\pi_{+} R\left(E+i 0, H_{0}\right) \beta_{1} \pi_{-}$, where $\pi_{ \pm}(x)$ is a smooth bounded function with support in $\Pi_{ \pm}$. This is written in the integral form

$$
\pi_{+} R\left(E+i 0 ; H_{0}\right) \beta_{1} \pi_{-}=i \int_{0}^{\infty} e^{i t E} \pi_{+} \exp \left(-i t H_{0}\right) \beta_{1} \pi_{-} d t
$$

and the integrand operator $\pi_{+} \exp \left(-i t H_{0}\right) \beta_{1} \pi_{-}$has the kernel

$$
G(t, x, y)=(2 \pi)^{-2} \int \exp (i \psi(t, x, y, \xi)) \pi_{+}(x) \beta_{1}(\xi) \pi_{-}(y) d \xi
$$

where $\psi=(x-y) \cdot \xi-t|\xi|^{2}$. If $x, y$ and $\xi$ are in $\Pi_{+}, \Pi_{-}$and supp $\beta_{1}$ respectively, then we take $\delta>0$ so small that $(y-x) \cdot \xi \geq 0$, and hence

$$
\left|\nabla_{\xi} \psi\right|^{2}=|x-y-2 t \xi|^{2} \geq|x-y|^{2}+4 t^{2}|\xi|^{2} \geq c\left(t+|x|+|y|+|d|^{\sigma}\right)^{2}
$$

for $t>0$. This shows by repeated use of partial integral that

$$
\pi_{+} R\left(E+i 0 ; H_{0}\right) \beta_{1} \pi_{-}=\tilde{r}_{N}
$$


for any $N \gg 1$, and we can put

$$
e^{i \gamma(x ;-\omega)}\left[H_{0}, j\right] R\left(E+i 0 ; H_{0}\right) \beta_{1} e^{-i \alpha \gamma(x ;-\omega)} T_{2}=\tilde{r}_{N}
$$

for the remainder term on the right side of (2.21). This means that the free particle starting from $\operatorname{supp} T_{2}$ at $t=0$ never passes over $\operatorname{supp} \nabla j$ for $t>0$. Thus we can approximate $R(E+i 0 ; H(A)) T_{2}$ as

$$
j e^{i \alpha \gamma(x ;-\omega)} R\left(E+i 0 ; H_{0}\right) \beta_{1} e^{-i \alpha \gamma(x ;-\omega)} T_{2}+R(E+i 0 ; H(A)) \tilde{r}_{N} .
$$

Note that $\tilde{T}_{1}^{*} \beta_{1}=\tilde{r}_{N}$ and $\tilde{T}_{2}^{*} \beta_{1}=\tilde{r}_{N}$ for any $N \gg 1$. The symbol $\tilde{T}_{1}(x, \xi)$ has support in $\left\{|x|<6|d|^{\sigma}\right\}$ in the $x$ variables. Hence we see from Lemma 2.6 that $\left\|\tilde{T}_{1}^{*} R(E+i 0 ; H(A)) T_{2}\right\|=O\left(|d|^{-N}\right)+\left\|\tilde{T}_{1}^{*} R(E+i 0 ; H(A)) \tilde{r}_{N}\right\|=O\left(|d|^{-N / 2}\right)$.

A similar approximation (incoming approximation) is constructed for

$$
\tilde{T}_{2}^{*} R(E+i 0 ; H(A))=\left(R(E-i 0 ; H(A)) \tilde{T}_{2}\right)^{*} .
$$

Hence it follows again from Lemma 2.6 that

$$
\begin{aligned}
\left\|\tilde{T}_{2}^{*} R(E+i 0 ; H(A)) T_{2}\right\| & =O\left(|d|^{-N}\right)+\left\|\tilde{T}_{2}^{*} R(E+i 0 ; H(A)) \tilde{r}_{N}\right\| \\
& =O\left(|d|^{-N}\right)+\left\|\tilde{r}_{N} R(E+i 0 ; H(A)) \tilde{r}_{N}\right\|=O\left(|d|^{-N}\right) .
\end{aligned}
$$

The same argument applies to the other operators and the proof is complete.

We obtain the following lemma as an immediate consequence of Lemma 2.5 .

Lemma 2.7. Let $\chi_{1 d}(x)=\chi\left(|x| / 3|d|^{\sigma}\right)$. Then

$$
f(\omega \rightarrow \tilde{\omega} ; E)=(i c(E) / 4 \pi)\left(R(E+i 0 ; H(A)) T_{1} \varphi_{0}(\omega, E), \tilde{T}_{1} \varphi_{0}(\tilde{\omega}, E)\right)+o(1)
$$

as $|d| \rightarrow \infty$, where $T_{1}$ acts as

$$
\begin{aligned}
& T_{1} \varphi_{0}(\omega, E)=e^{i \alpha \gamma(x ; \omega)} \chi_{1 d}\left[H_{0}, j_{0}\right] \varphi_{0}(\omega, E) \\
& \text { on } \varphi_{0}(\omega, E)=\varphi_{0}(x ; \omega, E) \text {, and } \tilde{T}_{1} \text { acts as } \\
& \tilde{T}_{1} \varphi_{0}(\tilde{\omega}, E)=e^{i \alpha \gamma(x ;-\tilde{\omega})} \chi_{1 d}\left[H_{0}, \tilde{j}_{0}\right] \varphi_{0}(\tilde{\omega}, E) \\
& \text { on } \varphi_{0}(\tilde{\omega}, E) \text {. }
\end{aligned}
$$




\section{§3. Proof of Theorem 1.1: Reduction to Two Lemmas}

In this section we prove Theorem 1.1. The proof is done by reduction to two lemmas. Throughout the section we always assume that the assumption in the theorem is fulfilled. In particular, $\omega$ and $\tilde{\omega}$ satisfy $\omega \neq \tilde{\omega}, \omega \neq \pm \hat{d}$ and $\tilde{\omega} \neq \pm \hat{d}$.

We first recall the notation. Let $b_{j} \in C_{0}^{\infty}\left(\boldsymbol{R}^{2}\right), 1 \leq j \leq 2$, be two given magnetic fields with total flux $\alpha_{j}$. For brevity, we assume that $b_{j}$ has support in $\{|x|<1\}$. Then it follows from Lemma 2.1 that there exists a smooth magnetic potential $A_{j}(x)$ associated with $b_{j}$ such that

$$
A_{j}(x)=\alpha_{j}\left(-x_{2} /|x|^{2}, x_{1} /|x|^{2}\right)
$$

for $|x|>2$. For these potentials, we define $H_{d}$ as

$$
H_{d}=H\left(A_{1}+A_{2 d}\right)=\left(-i \nabla-A_{1}-A_{2 d}\right)^{2}, \quad A_{2 d}(x)=A_{2}(x-d),
$$

with $d \in \boldsymbol{R}^{2},|d| \gg 1$. We denote by $f_{j}(\omega \rightarrow \tilde{\omega} ; E)$ and $f_{d}(\omega \rightarrow \tilde{\omega} ; E)$ the scattering amplitude for the pairs $\left(H\left(A_{j}\right), H_{0}\right)$ and $\left(H_{d}, H_{0}\right)$ respectively.

We still fix $0<\sigma \ll 1$ small enough as in Section 2. Let $j_{0}$ and $\tilde{j}_{0}$ be as in (2.7) and (2.9) respectively, where $R$ is taken as $R=|d|^{\sigma}$. We define the following three operators:

$$
\begin{aligned}
J_{0 d} & =j_{0} j_{0 d} \exp \left(i \alpha_{1} \gamma(x ; \omega)\right) \exp \left(i \alpha_{2} \gamma(x-d ; \omega)\right) \beta_{0}, \\
J_{1} & =j_{0} \exp \left(-i \alpha_{1} \gamma(x ; \omega)\right) \beta_{0}, \quad J_{1 d}=j_{0 d} \exp \left(-i \alpha_{2} \gamma(x-d ; \omega)\right) \beta_{0},
\end{aligned}
$$

where $j_{0 d}(x)=j_{0}(x-d)$ and $\beta_{0}=\beta_{0}\left(D_{x}\right)$. Then $W_{-}\left(H_{d}, H_{0}\right)$ is decomposed into

$$
W_{-}\left(H_{d}, H_{0}\right) \beta_{0}^{3}=W_{-}\left(H_{d}, H_{0} ; J_{0 d}\right) W_{-}\left(H_{0}, H_{0} ; J_{1}\right) W_{-}\left(H_{0}, H_{0} ; J_{1 d}\right) .
$$

By Lemma 2.2, the last operator on the right side equals $W_{-}\left(H_{0}, H_{0} ; I\right)$ with $I=j_{0} e^{-i \alpha_{2} \gamma(x ; \omega)} \beta_{0}$, and hence it is realized as

$$
F W_{-}\left(H_{0}, H_{0} ; J_{1 d}\right) F^{*}=e^{-i \alpha_{2} \gamma(-\theta ; \omega)} \beta_{0}(\sqrt{\lambda} \theta) \times
$$

on $L^{2}((0, \infty) ; d \lambda) \otimes L^{2}\left(S^{1}\right)$. A similar relation is true for $W_{+}\left(H_{d}, H_{0}\right)$ under natural modification of notation. We have

$$
W_{+}\left(H_{d}, H_{0}\right) \tilde{\beta}_{0}^{3}=W_{+}\left(H_{d}, H_{0} ; \tilde{J}_{0 d}\right) W_{+}\left(H_{0}, H_{0} ; \tilde{J}_{1}\right) W_{+}\left(H_{0}, H_{0} ; \tilde{J}_{1 d}\right),
$$

where

$$
\tilde{J}_{0 d}=\tilde{j}_{0} \tilde{j}_{0 d} \exp \left(i \alpha_{1} \gamma(x ;-\tilde{\omega})\right) \exp \left(i \alpha_{2} \gamma(x-d ;-\tilde{\omega})\right) \tilde{\beta}_{0}
$$


and

$$
\tilde{J}_{1}=\tilde{j}_{0} \exp \left(-i \alpha_{1} \gamma(x ;-\tilde{\omega})\right) \tilde{\beta}_{0}, \quad \tilde{J}_{1 d}=\tilde{j}_{0 d} \exp \left(-i \alpha_{2} \gamma(x-d ;-\tilde{\omega})\right) \tilde{\beta}_{0} .
$$

The scattering amplitude $f_{d}=f_{d}(\omega \rightarrow \tilde{\omega} ; E)$ behaves like

$$
f_{d}=(i c(E) / 4 \pi)\left(R\left(E+i 0 ; H_{d}\right) T_{d} \varphi_{0}(\omega, E), \tilde{T}_{d} \varphi_{0}(\tilde{\omega}, E)\right)+o(1)
$$

as $|d| \rightarrow \infty$, where

$$
T_{d}=H_{d} J_{0 d}-J_{0 d} H_{0}, \quad \tilde{T}_{d}=H_{d} \tilde{J}_{0 d}-\tilde{J}_{0 d} H_{0} .
$$

This is obtained by the same argument as used to derive (2.17). The two operators $T_{d}$ and $\tilde{T}_{d}$ are calculated as

$$
\begin{aligned}
& T_{d}=\exp \left(i \alpha_{1} \gamma(x ; \omega)\right) \exp \left(i \alpha_{2} \gamma(x-d ; \omega)\right)\left[H_{0}, j_{0} j_{0 d}\right] \beta_{0}, \\
& \tilde{T}_{d}=\exp \left(i \alpha_{1} \gamma(x ;-\tilde{\omega})\right) \exp \left(i \alpha_{2} \gamma(x-d ;-\tilde{\omega})\right)\left[H_{0}, \tilde{j}_{0} \tilde{j}_{0 d}\right] \tilde{\beta}_{0} .
\end{aligned}
$$

We now formulate the two lemmas on which the proof of Theorem 1.1 is based. We write $\left\|Q_{d}\right\| \simeq O\left(|d|^{\nu}\right)$, if $Q_{d}: L^{2} \rightarrow L^{2}$ obeys $\left\|Q_{d}\right\| \leq c_{\varepsilon}|d|^{\nu+\varepsilon}$ for any $\varepsilon>0$.

Lemma 3.1. Let $r_{L}$ be the multiplication defined by (2.18). Define $\chi_{1 d}(x)$ as in Lemma 2.7 and $\chi_{2 d}(x)$ as $\chi_{2 d}=\chi_{1 d}(x-d)=\chi\left(|x-d| / 3|d|^{\sigma}\right)$. If $L \gg 1$, then

(1) $\left\|r_{L} R\left(E+i 0 ; H_{d}\right) \chi_{1 d}\right\|+\left\|r_{L} R\left(E+i 0 ; H_{d}\right) \chi_{2 d}\right\|=O\left(|d|^{-L / 2}\right)$,

(2) $\left\|r_{L} R\left(E+i 0 ; H_{d}\right) r_{L}\right\|=O\left(|d|^{-L}\right)$.

Lemma 3.2. Let $\chi_{j d}, 1 \leq j \leq 2$, be as above. Then

$$
\begin{gathered}
\left\|\chi_{1 d} R\left(E+i 0 ; H_{d}\right) \chi_{2 d}\right\| \simeq O\left(|d|^{-1 / 2+4 \sigma}\right) \\
\left\|\chi_{1 d}\left(R\left(E+i 0 ; H_{d}\right)-R\left(E+i 0 ; H\left(A_{1}\right)\right)\right) \chi_{1 d}\right\| \simeq O\left(|d|^{-1+7 \sigma}\right), \\
\left\|\chi_{2 d}\left(R\left(E+i 0 ; H_{d}\right)-R\left(E+i 0 ; H\left(A_{2, d}\right)\right)\right) \chi_{2 d}\right\| \simeq O\left(|d|^{-1+7 \sigma}\right) .
\end{gathered}
$$

Remark 3.1. The lemmas above remain true for $R\left(E-i 0 ; H_{d}\right)$. Thus Lemma 3.1 shows

$$
\left\|\chi_{1 d} R\left(E+i 0 ; H_{d}\right) r_{L}\right\|=O\left(|d|^{-L / 2}\right)
$$

by adjoint. We often use such an immediate consequence without further references. 
We proceed to the proof of Theorem 1.1, accepting Lemmas 3.1 and 3.2 as proved. These lemmas are proved in Section 5 . We calculate $T_{d}$ as

$$
T_{d}=\exp \left(i \theta_{d}(x ; \omega)\right)\left(\left[H_{0}, j_{0}\right] j_{0 d}+j_{0}\left[H_{0}, j_{0 d}\right]\right) \beta_{0},
$$

where

$$
\theta_{d}(x ; \omega)=\alpha_{1} \gamma(x ; \omega)+\alpha_{2} \gamma(x-d ; \omega) .
$$

Recall that $j_{0}$ has support in $\Sigma\left(|d|^{\sigma}, \omega, \delta\right)$ and $j_{0}=1$ on $\Sigma\left(2|d|^{\sigma}, \omega, 2 \delta\right)$. By assumption, $\omega \neq \pm \hat{d}$. Hence we can choose $\delta$ so small that $j_{0 d}=1$ on supp $\chi_{1 d}$ and $j_{0}=1$ on $\operatorname{supp} \chi_{2 d}$, so that $T_{d}$ is decomposed into the sum of four operators

$$
T_{d}=T_{1 d}+T_{2 d}+T_{3 d}+T_{4 d}
$$

where

$$
\begin{aligned}
& T_{1 d}=\exp \left(i \theta_{d}(x ; \omega)\right) \chi_{1 d}\left[H_{0}, j_{0}\right] \beta_{0}, \\
& T_{2 d}=\exp \left(i \theta_{d}(x ; \omega)\right) \chi_{2 d}\left[H_{0}, j_{0 d}\right] \beta_{0}, \\
& T_{3 d}=\exp \left(i \theta_{d}(x ; \omega)\right)\left(1-\chi_{1 d}\right)\left[H_{0}, j_{0}\right] j_{0 d} \beta_{0}, \\
& T_{4 d}=\exp \left(i \theta_{d}(x ; \omega)\right)\left(1-\chi_{2 d}\right) j_{0}\left[H_{0}, j_{0 d}\right] \beta_{0} .
\end{aligned}
$$

Similarly we have the decomposition

$$
\begin{aligned}
& \tilde{T}_{1 d}=\exp \left(i \theta_{d}(x ;-\tilde{\omega})\right) \chi_{1 d}\left[H_{0}, \tilde{j}_{0}\right] \tilde{\beta}_{0}, \\
& \tilde{T}_{2 d}=\exp \left(i \theta_{d}(x ;-\tilde{\omega})\right) \chi_{2 d}\left[H_{0}, \tilde{j}_{0 d}\right] \tilde{\beta}_{0}, \\
& \tilde{T}_{3 d}=\exp \left(i \theta_{d}(x ;-\tilde{\omega})\right)\left(1-\chi_{1 d}\right)\left[H_{0}, \tilde{j}_{0}\right] \tilde{j}_{0 d} \tilde{\beta}_{0}, \\
& \tilde{T}_{4 d}=\exp \left(i \theta_{d}(x ;-\tilde{\omega})\right)\left(1-\chi_{2 d}\right) \tilde{j}_{0}\left[H_{0}, \tilde{j}_{0 d}\right] \tilde{\beta}_{0}
\end{aligned}
$$

for $\tilde{T}_{d}$. We now set

$$
\gamma_{j k}(d)=(i c(E) / 4 \pi)\left(R\left(E+i 0 ; H_{d}\right) T_{j d} \varphi_{0}(\omega, E), \tilde{T}_{k d} \varphi_{0}(\tilde{\omega}, E)\right)
$$

for $1 \leq j, k \leq 4$, and we assert that $\gamma_{j k}(d)=o(1)$ for $j \neq k$, and that

$$
\begin{gathered}
\gamma_{33}(d)=o(1), \quad \gamma_{44}(d)=o(1), \\
\gamma_{11}(d)=\exp \left(i \alpha_{2} \tau(-d ; \omega, \tilde{\omega})\right) f_{1}(\omega \rightarrow \tilde{\omega} ; E)+o(1), \\
\gamma_{22}(d)=\exp \left(i \alpha_{1} \tau(d ; \omega, \tilde{\omega})\right) f_{2, d}(\omega \rightarrow \tilde{\omega} ; E)+o(1)
\end{gathered}
$$

for $\tau(x ; \omega, \tilde{\omega})$ as in the theorem. If this is established, the proof is complete. 
First it immediately follows from Lemma 3.2 that $\gamma_{12}(d)=o(1)$ and $\gamma_{21}(d)=o(1)$. We consider $\gamma_{11}(d)$. By Lemma 3.2 again, we have

$$
\gamma_{11}(d)=(i c(E) / 4 \pi)\left(R\left(E+i 0 ; H\left(A_{1}\right)\right) T_{1 d} \varphi_{0}(\omega, E), \tilde{T}_{1 d} \varphi_{0}(\tilde{\omega}, E)\right)+o(1) .
$$

The operator $T_{1 d}$ acts as

$$
T_{1 d} \varphi_{0}(\omega, E)=\exp \left(i \theta_{d}(x ; \omega)\right) \chi_{1 d}\left[H_{0}, j_{0}\right] \varphi_{0}(\omega, E)
$$

on $\varphi_{0}(\omega, E)=\exp (i \sqrt{E} x \cdot \omega)$. Similarly

$$
\tilde{T}_{1 d} \varphi_{0}(\tilde{\omega}, E)=\exp \left(i \theta_{d}(x ;-\tilde{\omega})\right) \chi_{1 d}\left[H_{0}, \tilde{j}_{0}\right] \varphi_{0}(\tilde{\omega}, E) .
$$

Since $\gamma(x-d ; \omega)=\gamma(-d ; \omega)+O\left(|d|^{-1+\sigma}\right)$ on $\operatorname{supp} \chi_{1 d}, \exp \left(i \theta_{d}(x ; \omega)\right)$ behaves like

$$
\exp \left(i \theta_{d}(x ; \omega)\right)=\exp \left(i \alpha_{2} \gamma(-d ; \omega)\right) \exp \left(i \alpha_{1} \gamma(x ; \omega)\right)+O\left(|d|^{-1+\sigma}\right)
$$

on supp $\chi_{1 d}$. Similarly

$$
\exp \left(i \theta_{d}(x ;-\tilde{\omega})\right)=\exp \left(i \alpha_{2} \gamma(-d ;-\tilde{\omega})\right) \exp \left(i \alpha_{1} \gamma(x ;-\tilde{\omega})\right)+O\left(|d|^{-1+\sigma}\right) .
$$

These relations, together with Lemma 2.7, imply (3.3). A similar argument applies to $\gamma_{22}(d)$ and we get (3.4). To prove the assertion for the other terms, we show the following lemma.

Lemma 3.3. Let $T_{k d}, \tilde{T}_{k d}, 1 \leq k \leq 4$, be as above. Then

$$
\left\|\tilde{T}_{k d}^{*} R\left(E+i 0 ; H_{d}\right) T_{3 d}\right\|+\left\|T_{k d}^{*} R\left(E-i 0 ; H_{d}\right) \tilde{T}_{3 d}\right\|=O\left(|d|^{-N}\right)
$$

for any $N \gg 1$. A similar bound holds true for $T_{4 d}$ and $\tilde{T}_{4 d}$.

Proof. We construct an outgoing approximation for $R\left(E+i 0 ; H_{d}\right) T_{3 d}$. The construction uses the same idea as in the proof of Lemma 2.5. We use the notation $j$ and $\beta_{1}$ there, and we recall that $j$ satisfies $\operatorname{supp} j \subset \Sigma\left(|d|^{\sigma},-\omega, \delta\right)$ and $j=1$ on $\Sigma\left(2|d|^{\sigma},-\omega, 2 \delta\right)$. Hence $j T_{3 d}=T_{3 d}$. The assumption that $\omega \neq \pm \hat{d}$ is important. If $\omega \neq \hat{d}$, then we can take $\delta>0$ so small that $j_{d} T_{3 d}=T_{3 d}$ for $j_{d}(x)=j(x-d)$ also. We now write

$$
\theta(x)=\theta_{d}(x ;-\omega)=\alpha_{1} \gamma(x ;-\omega)+\alpha_{2} \gamma(x-d ;-\omega) .
$$

Then it follows from (2.6) that

$$
\begin{aligned}
\left(H_{d}-E\right) j j_{d} \exp (i \theta(x)) & =\exp (i \theta(x))\left(H_{0}-E\right) j j_{d} \\
& =\exp (i \theta(x))\left(j j_{d}\left(H_{0}-E\right)+\left[H_{0}, j j_{d}\right]\right)
\end{aligned}
$$


and this yields

$$
\begin{aligned}
& \left(H_{d}-E\right) j j_{d} e^{i \theta(x)} R\left(E+i 0 ; H_{0}\right) \beta_{1} e^{-i \theta(x)} T_{3 d} \\
& \quad=T_{3 d}+\tilde{r}_{N}+e^{i \theta(x)}\left[H_{0}, j j_{d}\right] R\left(E+i 0 ; H_{0}\right) \beta_{1} e^{-i \theta(x)} T_{3 d}
\end{aligned}
$$

for any $N \gg 1$, where $\tilde{r}_{N}$ is a bounded operator having the property (2.20). Since $\omega \neq \hat{d}$, the free particle starting from $(y, \xi) \in \operatorname{supp} T_{3 d}$ at $t=0$ never passes over $\operatorname{supp} \nabla j$ and $\operatorname{supp} \nabla j_{d}$ for $t>0$. In fact, if $\omega \neq \hat{d}$, then we can show after a simple consideration that

$$
|x-y-2 t \xi|^{2}=|x|^{2}+|y+2 t \xi|^{2}-2 t x \cdot(y+2 t \xi) \geq c\left(t+|x|+|y|+|d|^{\sigma}\right)^{2}
$$

for $x \in \operatorname{supp} \nabla j_{d}$. Hence we can put

$$
e^{i \theta(x)}\left[H_{0}, j j_{d}\right] R\left(E+i 0 ; H_{0}\right) \beta_{1} e^{-i \theta(x)} T_{3 d}=\tilde{r}_{N} .
$$

Thus $R\left(E+i 0 ; H_{d}\right) T_{3 d}$ is approximated by

$$
j j_{d} e^{i \theta(x)} R\left(E+i 0 ; H_{0}\right) \beta_{1} e^{-i \theta(x)} T_{3 d}+R\left(E+i 0 ; H_{d}\right) \tilde{r}_{N} .
$$

The operators $R\left(E+i 0 ; H_{d}\right) T_{4 d}$ and $R\left(E-i 0 ; H_{d}\right) \tilde{T}_{k d}, 3 \leq k \leq 4$, have similar approximations. If we use Lemma 3.1 (see Remark 3.1), the lemma is obtained by repeating almost the same argument as in the proof of Lemma 2.5.

We turn back to the proof of Theorem 1.1. The assertion for the remaining terms follows as an immediate consequence of Lemma 3.3. Thus the proof of Theorem 1.1 is now complete.

\section{$\S 4 . \quad$ Auxiliary Operators}

The free Hamiltonian $H_{0}$ is not necessarily a good approximation to $H_{d}$, because $H_{d}-H_{0}$ is a perturbation of long-range class as already stated. In this section we define several auxiliary operators to approximate $H_{d}$. These operators play an important role in proving Lemmas 3.1 and 3.2.

We fix $0<\sigma_{1}, \sigma_{2} \ll 1$ small enough and define the following two sets

$$
\Pi_{1 d}=\left\{|x|<C|d|^{\sigma_{1}}\right\} \cup\left\{|x| \geq C|d|^{\sigma_{1}}, \quad|\hat{x}+\hat{d}|<|d|^{-\sigma_{1} / 2}\right\},
$$

$$
\Pi_{2 d}=\left\{|x-d|<C|d|^{\sigma_{2}}\right\} \cup\left\{|x-d| \geq C|d|^{\sigma_{2}}, \quad|(\widehat{x-d})-\hat{d}|<|d|^{-\sigma_{2} / 2}\right\}
$$

for some $C \gg 1$, where $\hat{x}=x /|x|$. These two sets are disjoint with each other for $|d| \gg 1$. Let $\zeta_{j d} \in C^{\infty}(\boldsymbol{R}), 1 \leq j \leq 2$, be a real periodic function with 
period $2 \pi$ such that $\zeta_{j d}(s)=\alpha_{j} s$ on the interval $\left(|d|^{-\sigma_{j} / 2}, 2 \pi-|d|^{-\sigma_{j} / 2}\right)$ and it satisfies $\left|(d / d s)^{l} \zeta_{j d}(s)\right| \leq C_{l}|d|^{l \sigma_{j} / 2}$ for $C_{l}>0$ independent of $|d| \gg 1$, where $\alpha_{j}$ is the flux of field $b_{j}$.

We define a smooth real function $\eta_{1 d}$ by $\eta_{1 d}(x)=0$ for $|x|<|d|^{\sigma_{1}} / 2$ and by

$$
\eta_{1 d}(x)=\zeta_{1 d}(\gamma(x ;-\hat{d}))
$$

for $|x|>|d|^{\sigma_{1}}$. We may assume that $\eta_{1 d}$ satisfies

$$
\left|\partial_{x}^{\beta} \eta_{1 d}(x)\right| \leq C_{\beta}|d|^{|\beta| \sigma_{1} / 2}|x|^{-|\beta|} \leq \tilde{C}_{\beta}\langle x\rangle^{-|\beta| / 2}
$$

uniformly in $d$. By (4.2), we have

$$
\nabla \eta_{1 d}(x)=\zeta_{1 d}^{\prime}(\gamma(x ;-\hat{d})) \nabla \gamma(x ;-\hat{d})=\zeta_{1 d}^{\prime}(\gamma(x ;-\hat{d}))\left(-x_{2} /|x|^{2}, x_{1} /|x|^{2}\right)
$$

and hence

$$
\nabla \eta_{1 d}(x)=\alpha_{1}\left(-x_{2} /|x|^{2}, x_{1} /|x|^{2}\right)
$$

for $x \in \Pi_{1 d}^{\mathrm{c}}, \Pi_{1 d}^{\mathrm{c}}$ being the complement of $\Pi_{1 d}$. Similarly we define $\eta_{2 d}$ by

$$
\eta_{2 d}(x)=\zeta_{2 d}(\gamma(x-d ; \hat{d}))
$$

for $|x-d|>|d|^{\sigma_{2}}$ and by $\eta_{2 d}(x)=0$ for $|x-d|<|d|^{\sigma_{2}} / 2$.

We set $p_{1 d}(x)=\exp \left(i \eta_{1 d}(x)\right)$ and $q_{1 d}(x)=1 / p_{1 d}(x)$. By (4.3), we have

$$
\left|\partial_{x}^{\beta} p_{1 d}(x)\right|+\left|\partial_{x}^{\beta} q_{1 d}(x)\right| \leq C_{\beta}\langle x\rangle^{-|\beta| / 2}
$$

uniformly in $d$. If $x \in \Pi_{1 d}^{\mathrm{c}}$, then

$$
p_{1 d}(x)=\exp \left(i \alpha_{1} \gamma(x ;-\hat{d})\right), \quad q_{1 d}(x)=\exp \left(-i \alpha_{1} \gamma(x ;-\hat{d})\right) .
$$

Similarly we define $p_{2 d}(x)=\exp \left(i \eta_{2 d}(x)\right)$ and $q_{2 d}(x)=1 / p_{2 d}(x)$. Then

$$
\left|\partial_{x}^{\beta} p_{2 d}(x)\right|+\left|\partial_{x}^{\beta} q_{2 d}(x)\right| \leq C_{\beta}\langle x-d\rangle^{-|\beta| / 2}
$$

and

$$
p_{2 d}(x)=\exp \left(i \alpha_{2} \gamma(x-d ; \hat{d})\right), \quad q_{2 d}(x)=\exp \left(-i \alpha_{2} \gamma(x-d ; \hat{d})\right)
$$

for $x \in \Pi_{2 d}^{\mathrm{c}}$. 
We now introduce the following three operators

$$
\begin{aligned}
& K_{0 d}=p_{1 d} p_{2 d} H_{0} q_{2 d} q_{1 d}=H\left(\nabla \eta_{1 d}+\nabla \eta_{2 d}\right), \\
& K_{1 d}=p_{2 d} H\left(A_{1}\right) q_{2 d}=H\left(A_{1}+\nabla \eta_{2 d}\right), \\
& K_{2 d}=p_{1 d} H\left(A_{2 d}\right) q_{1 d}=H\left(\nabla \eta_{1 d}+A_{2 d}\right)
\end{aligned}
$$

as auxiliary operators. We consider the difference $W_{1 d}=K_{1 d}-K_{0 d}$. It follows from (3.1) and (4.5) that $\nabla \eta_{1 d}=A_{1}$ on $\Pi_{1 d}^{\mathrm{c}}$, and hence $W_{1 d}=0$ there. Similarly we have

$$
H_{d}-K_{2 d}=H\left(A_{1}+A_{2 d}\right)-K_{2 d}=0
$$

on $\Pi_{1 d}^{\mathrm{c}}$. Since $A_{2 d}(x)=A_{2}(x-d)=\nabla \eta_{2 d}$ on $\Pi_{1 d}$, we also have $W_{1 d}=H_{d}-K_{2 d}$ on $\Pi_{1 d}$. A similar argument applies to $W_{2 d}=K_{2 d}-K_{0 d}$. We can obtain the relations

$$
H_{d}=K_{1 d}+W_{2 d}, \quad H_{d}=K_{2 d}+W_{1 d} .
$$

The difference $W_{j d}$ is a differential operator of first order, and the coefficients have support in $\Pi_{j d}$. For example, $W_{1 d}$ takes the form

$$
W_{1 d}=2 i e_{1 d}(x) \cdot \nabla+e_{0 d}(x) .
$$

By (4.3) and (4.4), we see that $e_{1 d}$ and $e_{0 d}$ satisfy

$$
e_{1 d}(x)=\left(\alpha_{1}-\zeta_{1 d}^{\prime}(\gamma)\right) \nabla \gamma=O\left(|d|^{\sigma_{1} / 2}\right)|x|^{-1}
$$

with $\gamma=\gamma(x ;-\hat{d})$, and

$$
e_{0 d}(x)=O\left(|d|^{\sigma_{1}}\right)|x|^{-2}
$$

for $|x|>|d|^{\sigma_{1}}$. By (4.6), we have

$$
\left|\partial_{x}^{\beta} e_{0 d}(x)\right|+\left|\partial_{x}^{\beta} e_{1 d}(x)\right| \leq C_{\beta}\langle x\rangle^{-|\beta| / 2}
$$

uniformly in $d$. The operator $W_{2 d}$ has similar properties with natural modification. It should be noted that the coefficients are all bounded uniformly in $d$.

\section{$\S 5 . \quad$ Proof of Lemmas 3.1 and 3.2}

In this section we prove Lemmas 3.1 and 3.2. Throughout the section, $\sigma_{1}$ and $\sigma_{2}$ are fixed as $\sigma_{1}=\sigma_{2}=\sigma$, and the set $\Pi_{j d}, 1 \leq j \leq 2$, and the auxiliary 
operators $K_{j d}, 0 \leq j \leq 2$, are defined with $\sigma_{1}=\sigma_{2}=\sigma$ as in the previous section. We use the notation $b_{1 d}(x)$ to denote the characteristic function of the set $\left\{|x|<C|d|^{\sigma}\right\}, C \gg 1$ being as in (4.1), and we set $b_{2 d}(x)=b_{1 d}(x-d)$.

5.1. As the first step, we prove the resolvent estimates for the auxiliary operators as a series of lemmas. Let $G_{d}(x, y ; E)$ be the Green kernel of $R(E+$ $\left.i 0 ; K_{0 d}\right)=p_{d} R\left(E+i 0 ; H_{0}\right) q_{d}$, where $p_{d}=p_{1 d} p_{2 d}$ and $q_{d}=q_{1 d} q_{2 d}$. The resolvent $R\left(E+i 0 ; H_{0}\right)$ has the kernel

$$
G_{0}(x, y ; E)=(i / 4) H_{0}^{(1)}(\sqrt{E}|x-y|),
$$

where $H_{0}^{(1)}(z)$ is the Hankel function of first kind and order zero. As is well known, $H_{0}^{(1)}(z)$ behaves like

$$
H_{0}^{(1)}(z)=(2 / \pi)^{1 / 2} \exp (i(z-\pi / 4)) z^{-1 / 2}\left(1+O\left(|z|^{-1}\right)\right)
$$

at infinity. Hence $G_{d}(x, y ; E)$ behaves like

$$
G_{d}=c_{0}(E) p_{d}(x) \exp (i \sqrt{E}|x-y|)|x-y|^{-1 / 2} q_{d}(y)\left(1+O\left(|x-y|^{-1}\right)\right)
$$

as $|x-y| \rightarrow \infty$, where $c_{0}(E)=(1 / 8 \pi)^{1 / 2} \exp (i \pi / 4) E^{-1 / 4}$.

Lemma 5.1. Let $b_{1 d}$ and $b_{2 d}$ be as above. Then

$$
\left\|b_{2 d} R\left(E+i 0 ; K_{0 d}\right) b_{1 d}\right\|=O\left(|d|^{-1 / 2+2 \sigma}\right) .
$$

Proof. The bound follows from (5.1) at once. We have only to evaluate the Hilbert-Schmidt norm of the operator.

Lemma 5.2. Let $r_{L}$ be the multiplication defined by (2.18). If $L \gg 1$ is large enough and if $\rho, 1 / 2<\rho<1$, is close enough to $1 / 2$, then

$$
\left\|\langle x\rangle^{\rho} W_{1 d} R\left(E+i 0 ; K_{0 d}\right) r_{L}\right\|=O\left(|d|^{-L / 2}\right) .
$$

Proof. We write $R\left(E+i 0 ; K_{0 d}\right)=p_{d} R\left(E+i 0 ; H_{0}\right) q_{d}$. It follows from (4.10), (4.11) and (4.12) with $\sigma_{1}=\sigma$ that $W_{1 d}$ takes the form

$$
W_{1 d}=O\left(|d|^{\sigma / 2}\right) \nabla \gamma \cdot \nabla+O\left(|d|^{\sigma}\right)|x|^{-2}
$$

in $\left\{x:|x|>|d|^{\sigma}\right\}$, where $\gamma=\gamma(x ;-\hat{d})$. The operator $\nabla \gamma \cdot \nabla$ is written as

$$
\nabla \gamma \cdot \nabla=|x|^{-2}\left(-x_{2} \partial_{1}+x_{1} \partial_{2}\right)=|x|^{-2} \partial / \partial \theta
$$


and $p_{d}$ satisfies

$$
\nabla p_{d}=O\left(|x|^{-1 / 2}\right)+O\left(|x-d|^{-1 / 2}\right)
$$

uniformly in $d$ by (4.6). Since $H_{0}$ and $\partial / \partial \theta$ commute each other and since

$$
\langle x\rangle^{-\rho} R\left(E+i 0 ; H_{0}\right) \nabla\langle x\rangle^{-\rho}: L^{2} \rightarrow L^{2}
$$

is bounded for $\rho>1 / 2$ by the principle of limiting absorption, the lemma is easily verified.

5.2. We further prove the two lemmas below on the resolvent estimates for the auxiliary operators. To prove the lemmas, we work in the phase space. Let $\left\{\beta_{ \pm}, \beta_{\infty}\right\}$ be a smooth nonnegative partition of unity over $\boldsymbol{R}_{\xi}^{2}$. The partition is normalized by

$$
\beta_{+}(\xi)+\beta_{-}(\xi)+\beta_{\infty}(\xi)=1
$$

and it has the following properties: $\operatorname{supp} \beta_{\infty} \subset\{|\xi|<E / 2$ or $|\xi|>2 E\}$ and

$$
\operatorname{supp} \beta_{ \pm} \subset\{E / 3<|\xi|<3 E, \pm \hat{\xi} \cdot \hat{d}>-1 / 4\} \text {. }
$$

\section{Lemma 5.3.}

$$
\left\|b_{2 d} R\left(E+i 0 ; K_{1 d}\right) b_{1 d}\right\|+\left\|b_{1 d} R\left(E+i 0 ; K_{2 d}\right) b_{2 d}\right\| \simeq O\left(|d|^{-1 / 2+3 \sigma}\right) .
$$

Lemma 5.4. Let $\rho>1 / 2$ be as in Lemma 5.2. If $L \gg 1$, then

$$
\left\|\langle x\rangle^{\rho} W_{1 d} R\left(E+i 0 ; K_{2 d}\right) r_{L}\right\|=O\left(|d|^{-L / 2}\right) .
$$

The approximations for $R\left(E+i 0 ; K_{j d}\right), 1 \leq j \leq 2$, play an important role in proving the above lemmas. Before going into the proof, we explain how to construct such approximations. This idea is repeatedly used in the future discussion as well as in the proof of Lemmas 5.3 and 5.4.

We now consider $W_{1 d}=K_{1 d}-K_{0 d}$. According to (5.2), it is decomposed into the sum of four operators

$$
W_{1 d}=g_{1 d}^{2} W_{1 d}+V_{\infty}\left(x, D_{x}\right)+V_{+}\left(x, D_{x}\right)+V_{-}\left(x, D_{x}\right),
$$

where $g_{1 d}(x)=\chi\left(|x| / M|d|^{\sigma}\right)$ for $M \gg 1$, and

$$
V_{ \pm}\left(x, D_{x}\right)=\left(1-g_{1 d}^{2}\right) W_{1 d} \beta_{ \pm}\left(D_{x}\right), \quad V_{\infty}\left(x, D_{x}\right)=\left(1-g_{1 d}^{2}\right) W_{1 d} \beta_{\infty}\left(D_{x}\right) .
$$


Since the coefficients of $W_{1 d}$ have support in $\Pi_{1 d}$ defined by (4.1) with $\sigma_{1}=\sigma$, $V_{+}(x, \xi)$ is supported in the set

$$
\left\{|x|>M|d|^{\sigma}, \quad|\hat{x}+\hat{d}|<|d|^{-\sigma / 2}, \quad E / 3<|\xi|<3 E, \quad \hat{\xi} \cdot \hat{d}>-1 / 4\right\}
$$

of the phase space, and $(x, \xi) \in \operatorname{supp} V_{+}$has the incoming property

$$
\hat{x} \cdot \hat{\xi}=-\hat{d} \cdot \hat{\xi}+(\hat{x}+\hat{d}) \cdot \hat{\xi}<1 / 3
$$

for $|d| \gg 1$. We construct an incoming approximation for

$$
V_{+} R\left(E+i 0 ; K_{1 d}\right)=\left(R\left(E-i 0 ; K_{1 d}\right) V_{+}^{*}\right)^{*} .
$$

The operator $V_{+}^{*}$ is expanded as $V_{+}^{*}=V_{+N}+\tilde{r}_{N}$ for any $N \gg 1$, where $\tilde{r}_{N}$ has the property $(2.20)$. The symbol $V_{+N}(x, \xi)$ has support in the same region as above and it satisfies

$$
\left|\partial_{x}^{\beta} \partial_{\xi}^{\gamma} V_{+N}(x, \xi)\right| \leq C_{N \beta \gamma}\left(|x|+|d|^{\sigma}\right)^{-|\beta| / 2}
$$

by (4.13). The construction uses almost the same way as in the proof of Lemmas 2.5 and 3.3. Let $j_{ \pm}(x)=j_{ \pm}(x ; d)$ be a smooth function such that $\partial_{x}^{\beta} j_{ \pm}(x)=$ $O\left(|x|^{-|\beta|}\right)$ uniformly in $d$ and

$$
\operatorname{supp} j_{ \pm} \subset \Sigma\left(|d|^{\sigma}, \pm \hat{d}, \delta\right), \quad j_{ \pm}=1 \text { on } \Sigma\left(2|d|^{\sigma}, \pm \hat{d}, 2 \delta\right) .
$$

Then $j_{+} V_{+N}=V_{+N}$ and we have

$$
\begin{aligned}
\left(K_{1 d}-E\right) j_{+} e^{i \alpha_{1} \gamma(x ; \hat{d})} & =p_{2 d}\left(H\left(A_{1}\right)-E\right) j_{+} e^{i \alpha_{1} \gamma(x ; \hat{d})} q_{2 d} \\
& =p_{2 d} e^{i \alpha_{1} \gamma(x ; \hat{d})}\left(H_{0}-E\right) j_{+} q_{2 d} \\
& =p_{2 d} e^{i \alpha_{1} \gamma(x ; \hat{d})}\left(j_{+}\left(H_{0}-E\right)+\left[H_{0}, j_{+}\right]\right) q_{2 d} .
\end{aligned}
$$

We can take $M \gg 1$ so large that the free particle reaching $(y, \xi) \in \operatorname{supp} V_{+}$at $t=0$ never passes over supp $\nabla j_{+}$for $t<0$. In fact, if $x \in \operatorname{supp} \nabla j_{+}$satisfies $|x| \leq 2|d|^{\sigma}$, then $|x| \leq 2|y| / M, M \gg 1$, and

$|x-y-2 t \xi|^{2} \geq|y-x|^{2}+2 t(1 / 3+2 / M)|y||\xi|+4 t^{2}|\xi|^{2} \geq c\left(|t|+|x|+|y|+|d|^{\sigma}\right)^{2}$

and if $x \in \operatorname{supp} \nabla j_{+}$satisfies $|x|>2|d|^{\sigma}$, then $y-x \sim(|y|+|x|) \hat{y}$, and

$$
|x-y-2 t \xi|^{2} \geq c\left(|t|+|x|+|y|+|d|^{\sigma}\right)^{2} .
$$

Let $\tilde{\beta}_{ \pm} \in C_{0}^{\infty}\left(\boldsymbol{R}^{2}\right)$ be a real symbol such that $\tilde{\beta}_{ \pm}(\xi)=1$ on $\operatorname{supp} \beta_{ \pm}$. We may assume that $\operatorname{supp} \tilde{\beta}_{ \pm}$is slightly wider than $\operatorname{supp} \beta_{ \pm}$. If we make use of the above relation, then it follows that

$$
\left(K_{1 d}-E\right) j_{+} e^{i \alpha_{1} \gamma(x ; \hat{d})} p_{2 d} R\left(E-i 0 ; H_{0}\right) \tilde{\beta}_{+} q_{2 d} e^{-i \alpha_{1} \gamma(x ; \hat{d})} V_{+N}=V_{+N}+\tilde{r}_{N} .
$$


Thus $R\left(E-i 0 ; K_{1 d}\right) V_{+}^{*}$ is represented as

$$
j_{+} e^{i \alpha_{1} \gamma(x ; \hat{d})} p_{2 d} R\left(E-i 0 ; H_{0}\right) \tilde{\beta}_{+} q_{2 d} e^{-i \alpha_{1} \gamma(x ; \hat{d})} V_{+N}+R\left(E-i 0 ; K_{1 d}\right) \tilde{r}_{N},
$$

so that $V_{+} R\left(E+i 0 ; K_{1 d}\right)$ is approximated as

$$
\left(j_{+} e^{i \alpha_{1} \gamma(x ; \hat{d})} p_{2 d} R\left(E-i 0 ; H_{0}\right) \tilde{\beta}_{+} q_{2 d} e^{-i \alpha_{1} \gamma(x ; \hat{d})} V_{+N}\right)^{*}+\tilde{r}_{N} R\left(E+i 0 ; K_{1 d}\right) .
$$

Next we consider $W_{2 d}=K_{2 d}-K_{0 d}$. It is decomposed into the sum of four operators

$$
W_{2 d}=g_{2 d}^{2} W_{2 d}+W_{\infty}\left(x, D_{x}\right)+W_{+}\left(x, D_{x}\right)+W_{-}\left(x, D_{x}\right),
$$

where $g_{2 d}(x)=\chi\left(|x-d| / M|d|^{\sigma}\right)$ for $M \gg 1$, and

$$
W_{ \pm}\left(x, D_{x}\right)=\left(1-g_{2 d}^{2}\right) W_{2 d} \beta_{ \pm}\left(D_{x}\right), \quad W_{\infty}\left(x, D_{x}\right)=\left(1-g_{2 d}^{2}\right) W_{2 d} \beta_{\infty}\left(D_{x}\right) .
$$

The symbol $W_{-}(x, \xi)$ has support in the set

$$
\left\{|x-d|>M|d|^{\sigma}, \quad|(\widehat{x-d})-\hat{d}|<|d|^{-\sigma / 2}, \quad E / 3<|\xi|<3 E, \quad \hat{\xi} \cdot \hat{d}<1 / 4\right\}
$$

and $(x, \xi) \in \operatorname{supp} W_{-}$satisfies $(\widehat{x-d}) \cdot \hat{\xi}<1 / 3$ and

$$
\hat{x} \cdot \hat{\xi}=\hat{d} \cdot \hat{\xi}+(\hat{x}-\hat{d}) \cdot \hat{\xi}<1 / 3 \text {. }
$$

Then the free particle reaching supp $W_{-}$at $t=0$ does not pass over $\operatorname{supp} j_{-}$ for $t<0$. Thus $W_{-} R\left(E+i 0 ; K_{1 d}\right)$ is approximated as

$$
\left(j_{-} e^{i \alpha_{1} \gamma(x ;-\hat{d})} p_{2 d} R\left(E-i 0 ; H_{0}\right) \tilde{\beta}_{-} q_{2 d} e^{-i \alpha_{1} \gamma(x ;-\hat{d})} W_{-N}\right)^{*}
$$

with $\tilde{r}_{N} R\left(E+i 0 ; K_{1 d}\right)$ as an error operator, where $W_{-N}(x, \xi)$ satisfies

$$
\left|\partial_{x}^{\beta} \partial_{\xi}^{\gamma} W_{-N}(x, \xi)\right| \leq C_{N \beta \gamma}\left(|x-d|+|d|^{\sigma}\right)^{-|\beta| / 2}
$$

and has support in the same region as above.

A similar argument applies to $R\left(E+i 0 ; K_{2 d}\right)$. For example, $W_{-} R(E+$ $\left.i 0 ; K_{2 d}\right)$ is approximated as

$$
\left(j_{-d} e^{i \alpha_{2} \gamma(x-d ;-\hat{d})} p_{1 d} R\left(E-i 0 ; H_{0}\right) \tilde{\beta}_{-} q_{1 d} e^{-i \alpha_{2} \gamma(x-d ;-\hat{d})} W_{-N}\right)^{*}
$$

with error operator $\tilde{r}_{N} R\left(E+i 0 ; K_{2 d}\right)$, where $j_{ \pm d}(x)=j_{ \pm}(x-d)$. 
Proof of Lemma 5.3. We consider only the first operator $b_{2 d} R\left(E+i 0 ; K_{1 d}\right)$ $\times b_{1 d}$ on the left side. Since $K_{1 d}=K_{0 d}+W_{1 d}$, the resolvent identity yields

$$
R\left(E+i 0 ; K_{1 d}\right)=R\left(E+i 0 ; K_{0 d}\right)-R\left(E+i 0 ; K_{0 d}\right) W_{1 d} R\left(E+i 0 ; K_{1 d}\right) .
$$

By Lemma 5.1, the first operator on the right side obeys

$$
\left\|b_{2 d} R\left(E+i 0 ; K_{0 d}\right) b_{1 d}\right\|=O\left(|d|^{-1 / 2+2 \sigma}\right) .
$$

We decompose $W_{1 d}$ as in (5.3) to evaluate the second operator. We set

$$
G_{0}=b_{2 d} R\left(E+i 0 ; K_{0 d}\right) g_{1 d}^{2} W_{1 d} R\left(E+i 0 ; K_{1 d}\right) b_{1 d} .
$$

By the principle of limiting absorption,

$$
\langle x\rangle^{-\rho} R\left(E+i 0 ; K_{1 d}\right)\langle x\rangle^{-\rho}=p_{2 d}\langle x\rangle^{-\rho} R\left(E+i 0 ; H\left(A_{1}\right)\right)\langle x\rangle^{-\rho} q_{2 d}: L^{2} \rightarrow L^{2}
$$

is bounded for any $\rho>1 / 2$. This implies that $\left\|g_{1 d} R\left(E+i 0 ; K_{1 d}\right) b_{1 d}\right\| \simeq O\left(|d|^{\sigma}\right)$, and hence

$$
\left\|g_{1 d} \nabla R\left(E+i 0 ; K_{1 d}\right) b_{1 d}\right\| \simeq O\left(|d|^{\sigma}\right)
$$

by elliptic estimate. Since the coefficients of $W_{1 d}$ are all bounded uniformly in $d$, we have

$$
\left\|g_{1 d} W_{1 d} R\left(E+i 0 ; K_{1 d}\right) b_{1 d}\right\| \simeq O\left(|d|^{\sigma}\right) .
$$

Thus $G_{0}$ obeys $\left\|G_{0}\right\| \simeq O\left(|d|^{-1 / 2+3 \sigma}\right)$ by Lemma 5.1. The operator $V_{+} R(E+$ $\left.i 0 ; K_{1 d}\right)$ is approximated by $(5.6)$. The symbol $V_{+}(x, \xi)$ has support in the incoming region (5.4). If $M \gg 1$, then the free particle reaching $\operatorname{supp} V_{+}$at $t=0$ never passes over supp $b_{1 d}$ for $t<0$. This implies that

$$
V_{+} R\left(E+i 0 ; K_{1 d}\right) b_{1 d}=\tilde{r}_{N}+\tilde{r}_{N} R\left(E+i 0 ; K_{1 d}\right) b_{1 d}
$$

and hence we have

$$
\left\|b_{2 d} R\left(E+i 0 ; K_{0 d}\right) V_{+} R\left(E+i 0 ; K_{1 d}\right) b_{1 d}\right\|=O\left(|d|^{-N}\right)
$$

for any $N \gg 1$. The symbol $V_{-}(x, \xi)$ has support in the set

$$
\left\{|x|>M|d|^{\sigma}, \quad|\hat{x}+\hat{d}|<|d|^{-\sigma / 2}, \quad E / 3<|\xi|<3 E, \hat{\xi} \cdot \hat{d}<1 / 4\right\}
$$

and $(x, \xi) \in \operatorname{supp} V_{-}$has the outgoing property $\hat{x} \cdot \hat{\xi}>-1 / 3$. Hence the free particle starting from $\operatorname{supp} V_{-}$at $t=0$ does not pass over $\operatorname{supp} b_{2 d}$ for $t>0$. This shows that

$$
\begin{aligned}
b_{2 d} R\left(E+i 0 ; K_{0 d}\right) V_{-} & =b_{2 d} p_{d} R\left(E+i 0 ; H_{0}\right) \tilde{\beta}_{-} q_{d} V_{-}+b_{2 d} p_{d} R\left(E+i 0 ; H_{0}\right) \tilde{r}_{N} \\
& =\tilde{r}_{N}+b_{2 d} R\left(E+i 0 ; K_{0 d}\right) \tilde{r}_{N}
\end{aligned}
$$


for any $N \gg 1$, so that

$$
\left\|b_{2 d} R\left(E+i 0 ; K_{0 d}\right) V_{-} R\left(E+i 0 ; K_{1 d}\right) b_{1 d}\right\|=O\left(|d|^{-N}\right) .
$$

Finally we consider the operator

$$
G_{\infty}=b_{2 d} R\left(E+i 0 ; K_{0 d}\right) V_{\infty} R\left(E+i 0 ; K_{1 d}\right) b_{1 d}
$$

and prove $\left\|G_{\infty}\right\|=O\left(|d|^{-N}\right)$ by constructing an approximation for $V_{\infty} R(E+$ $\left.i 0 ; K_{1 d}\right)$. We follow the standard way used in constructing parametrices for elliptic operators. The symbol $V_{\infty}(x, \xi)$ has support in

$$
\left\{|x|>M|d|^{\sigma}, \quad|\hat{x}+\hat{d}|<|d|^{-\sigma / 2}, \quad|\xi|<E / 2 \text { or }|\xi|>2 E\right\}
$$

and $K_{1 d}(x, \xi)-E \sim|\xi|^{2}-E$ is invertible on $\operatorname{supp} V_{\infty}$. This enables us to construct the approximation in the form

$$
V_{\infty} R\left(E+i 0 ; K_{1 d}\right)=G_{N}\left(x, D_{x}\right)+\tilde{r}_{N} R\left(E+i 0 ; K_{1 d}\right)
$$

for any $N \gg 1$, where $G_{N}(x, \xi)$ takes the form

$$
G_{N}(x, \xi)=V_{\infty N}(x, \xi) /\left(K_{1 d}(x, \xi)-E\right) .
$$

The symbol $V_{\infty N}(x, \xi)$ has support in the same region as above and it satisfies

$$
\left|\partial_{x}^{\beta} \partial_{\xi}^{\gamma} V_{\infty N}(x, \xi)\right| \leq C_{N \beta \gamma}\left(|x|+|d|^{\sigma}\right)^{-|\beta| / 2} .
$$

If $M \gg 1$, then supp $V_{\infty N}$ and $\operatorname{supp} b_{1 d}$ do not intersect with each other. Hence we have

$$
V_{\infty} R\left(E+i 0 ; K_{1 d}\right) b_{1 d}=\tilde{r}_{N}+\tilde{r}_{N} R\left(E+i 0 ; K_{1 d}\right) b_{1 d} .
$$

This yields $\left\|G_{\infty}\right\|=O\left(|d|^{-N}\right)$. The proof of the lemma is now complete.

Proof of Lemma 5.4. The proof is based on the same idea as in the proof of Lemma 5.3. By the resolvent identity, we have

$$
R\left(E+i 0 ; K_{2 d}\right)=R\left(E+i 0 ; K_{0 d}\right)-R\left(E+i 0 ; K_{0 d}\right) W_{2 d} R\left(E+i 0 ; K_{2 d}\right) .
$$

By Lemma 5.2, the first operator obeys

$$
\left\|\langle x\rangle^{\rho} W_{1 d} R\left(E+i 0 ; K_{0 d}\right) r_{L}\right\|=O\left(|d|^{-L / 2}\right) .
$$

We decompose $W_{2 d}$ as in (5.7). The argument in the proof of Lemma 5.2 shows that 


$$
\left\|\langle x\rangle^{\rho} W_{1 d} R\left(E+i 0 ; K_{0 d}\right)\langle x\rangle^{-2}\right\|=O\left(|d|^{\nu}\right)
$$

for some $\nu>0$. Since $g_{2 d}(x)$ has support in $\left\{|x-d|<2 M|d|^{\sigma}\right\}$, it follows that

$$
\left\|\langle x\rangle^{\rho} W_{1 d} R\left(E+i 0 ; K_{0 d}\right) g_{2 d}\right\|=O\left(|d|^{\nu}\right)
$$

for another $\nu>0$, and hence we have

$$
\left\|\langle x\rangle^{\rho} W_{1 d} R\left(E+i 0 ; K_{0 d}\right) g_{2 d}^{2} W_{2 d} R\left(E+i 0 ; K_{2 d}\right) r_{L}\right\|=O\left(|d|^{-L / 2}\right) .
$$

The symbol $W_{+}(x, \xi)$ has support in the set

$$
\left\{|x-d|>M|d|^{\sigma}, \quad|(\widehat{x-d})-\hat{d}|<|d|^{-\sigma / 2}, \quad E / 3<|\xi|<3 E, \quad \widehat{\xi} \cdot \hat{d}>-1 / 4\right\}
$$

and the coefficients of $W_{1 d}$ have support in $\Pi_{1 d}$. Since $y-d \sim|y-d| \hat{d}$ for $(y, \xi) \in \operatorname{supp} W_{+}$and since $x-d \sim-|x-d| \hat{d}$ for $x \in \Pi_{1 d}$, it is easily seen that the free particle starting from supp $W_{+}$at $t=0$ does not pass over $\Pi_{1 d}$ for $t>0$. This yields that

$$
\langle x\rangle^{\rho} W_{1 d} R\left(E+i 0 ; K_{0 d}\right) W_{+}=\tilde{r}_{N}+\langle x\rangle^{\rho} W_{1 d} R\left(E+i 0 ; K_{0 d}\right) \tilde{r}_{N}
$$

for any $N \gg 1$, so that we obtain

$$
\left\|\langle x\rangle^{\rho} W_{1 d} R\left(E+i 0 ; K_{0 d}\right) W_{+} R\left(E+i 0 ; K_{2 d}\right) r_{L}\right\|=O\left(|d|^{-N}\right)
$$

by Lemma 5.2. The operator $W_{-} R\left(E+i 0 ; K_{2 d}\right)$ is approximated by $(5.9)$. If we note that $\langle x\rangle /\langle x-d\rangle=O(|d|)$, then it follows from Lemma 2.6 that

$$
\left\|\langle x\rangle^{2} W_{-} R\left(E+i 0 ; K_{2 d}\right) r_{L}\right\|=O\left(|d|^{-2 L / 3}\right)
$$

for $L \gg 1$. Thus we have

$$
\left\|\langle x\rangle^{\rho} W_{1 d} R\left(E+i 0 ; K_{0 d}\right) W_{-} R\left(E+i 0 ; K_{2 d}\right) r_{L}\right\|=O\left(|d|^{-L / 2}\right)
$$

by (5.10). If we use the same argument as in the proof of Lemma 5.3, we can construct the approximation for $W_{\infty} R\left(E+i 0 ; K_{2 d}\right)$ in the form

$$
W_{\infty} R\left(E+i 0 ; K_{2 d}\right) r_{L}=\tilde{r}_{L}+\tilde{r}_{N} R\left(E+i 0 ; K_{2 d}\right) r_{L}
$$

and hence we have

$$
\left\|\langle x\rangle^{\rho} W_{1 d} R\left(E+i 0 ; K_{0 d}\right) W_{\infty} R\left(E+i 0 ; K_{2 d}\right) r_{L}\right\|=O\left(|d|^{-L / 2}\right)
$$

by Lemma 5.2. This completes the proof.

5.3. We prove Lemmas 3.1 and 3.2 in question, accepting the following two propositions as proved. These propositions are proved in the next section. 
Proposition 5.1. Let $\pi_{j d}(x)$ be the characteristic function of $\Pi_{j d}$. Assume that $\rho>1 / 2$. If $L \gg 1$, then

$\left\|r_{L} R\left(E+i 0 ; H_{d}\right) \pi_{1 d}\langle x\rangle^{-\rho}\right\|+\left\|r_{L} R\left(E+i 0 ; H_{d}\right) \pi_{2 d}\langle x-d\rangle^{-\rho}\right\|=O\left(|d|^{-2 L / 3}\right)$.

\section{Proposition 5.2.}

$$
\left\|b_{2 d} R\left(E+i 0 ; H_{d}\right) b_{1 d}\right\| \simeq O\left(|d|^{3 \sigma}\right) .
$$

Proof of Lemma 3.1. Since $\left\{|x|<6|d|^{\sigma}\right\} \subset \Pi_{1 d}$, it is obvious from Proposition 5.1 that

$$
\left\|r_{L} R\left(E+i 0 ; H_{d}\right) \chi_{1 d}\right\|=O\left(|d|^{-L / 2}\right) .
$$

A similar bound is true for $\chi_{2 d}$ and (1) is established. We shall prove (2). Since $H_{d}=K_{2 d}+W_{1 d}$ by (4.9), we have

$$
R\left(E+i 0 ; H_{d}\right)=R\left(E+i 0 ; K_{2 d}\right)-R\left(E+i 0 ; H_{d}\right) W_{1 d} R\left(E+i 0 ; K_{2 d}\right)
$$

by the resolvent identity. We decompose $W_{1 d}=\left(\pi_{1 d}\langle x\rangle^{-\rho}\right)\left(\langle x\rangle^{\rho} W_{1 d}\right)$. Then it follows from Proposition 5.1 and Lemma 5.4 that

$$
\left\|r_{L} R\left(E+i 0 ; H_{d}\right) W_{1 d} R\left(E+i 0 ; K_{2 d}\right) r_{L}\right\|=O\left(|d|^{-L}\right) .
$$

This proves (2) and the proof is complete.

We proceed to the proof of Lemma 3.2. The outgoing approximation for the resolvent $R\left(E+i 0 ; H_{d}\right)$ is important to prove the lemma. We shall briefly explain how to construct such an approximation. The construction is based on the same idea as in the previous subsection, and we use the notation there. Let $j_{ \pm}$be as in $(5.5)$ and set $j_{ \pm d}(x)=j_{ \pm}(x-d)$ again. We consider $R\left(E+i 0 ; H_{d}\right) V_{-}$. Recall that $V_{-}(x, \xi)$ has support in the set

$$
\left\{|x|>M|d|^{\sigma}, \quad|\hat{x}+\hat{d}|<|d|^{-\sigma / 2}, \quad E / 3<|\xi|<3 E, \quad \hat{\xi} \cdot \hat{d}<1 / 4\right\} .
$$

Hence $V_{-}$satisfies $j_{+} V_{-}=V_{-}$. If we set

$$
\theta_{ \pm}(x)=\theta_{d}(x ; \pm \hat{d})=\alpha_{1} \gamma(x ; \pm \hat{d})+\alpha_{2} \gamma(x-d ; \pm \hat{d}),
$$

then $\nabla \theta_{+}=A_{1}(x)+A_{2 d}(x)$ on $\operatorname{supp} j_{+}$, and hence we have

$$
\begin{aligned}
\left(H_{d}-E\right) j_{+} \exp \left(i \theta_{+}(x)\right) & =\exp \left(i \theta_{+}(x)\right)\left(H_{0}-E\right) j_{+} \\
& =\exp \left(i \theta_{+}(x)\right)\left(j_{+}\left(H_{0}-E\right)+\left[H_{0}, j_{+}\right]\right) .
\end{aligned}
$$


This yields

$$
\begin{aligned}
& \left(H_{d}-E\right) j_{+} e^{i \theta_{+}(x)} R\left(E+i 0 ; H_{0}\right) \tilde{\beta}_{-} e^{-i \theta_{+}(x)} V_{-} \\
& \quad=V_{-}+\tilde{r}_{N}+e^{i \theta_{+}(x)}\left[H_{0}, j_{+}\right] R\left(E+i 0 ; H_{0}\right) \tilde{\beta}_{-} e^{-i \theta_{+}(x)} V_{-}
\end{aligned}
$$

for any $N \gg 1$. Since $(x, \xi) \in \operatorname{supp} V_{-}$has the outgoing property $\hat{x} \cdot \hat{\xi}>-1 / 3$, we can take $M \gg 1$ so large that the free particle starting from $\operatorname{supp} V_{-}$at $t=0$ does not pass over $\operatorname{supp} \nabla j_{+}$for $t>0$. Thus $R\left(E+i 0 ; H_{d}\right) V_{-}$can be approximated as

$$
j_{+} e^{i \theta_{+}(x)} R\left(E+i 0 ; H_{0}\right) \tilde{\beta}_{-} e^{-i \theta_{+}(x)} V_{-}+R\left(E+i 0 ; H_{d}\right) \tilde{r}_{N}
$$

Similarly we can construct the outgoing approximation

$$
j_{-d} e^{i \theta_{-}(x)} R\left(E+i 0 ; H_{0}\right) \tilde{\beta}_{+} e^{-i \theta_{-}(x)} W_{+}+R\left(E+i 0 ; H_{d}\right) \tilde{r}_{N}
$$

for $R\left(E+i 0 ; H_{d}\right) W_{+}$.

Proof of Lemma 3.2. The proof is based on the following three inequalities:

$$
\begin{aligned}
& \left\|\chi_{1 d}\left(R\left(E+i 0 ; H_{d}\right)-R\left(E+i 0 ; K_{1 d}\right)\right) \chi_{1 d}\right\| \\
& \quad \leq C_{\varepsilon}|d|^{-1 / 2+3 \sigma+\varepsilon}\left\|\chi_{1 d} R\left(E+i 0 ; H_{d}\right) g_{2 d}\right\|+C_{N}|d|^{-N}
\end{aligned}
$$

$$
\begin{aligned}
& \left\|\chi_{2 d}\left(R\left(E+i 0 ; H_{d}\right)-R\left(E+i 0 ; K_{2 d}\right)\right) \chi_{2 d}\right\| \\
& \quad \leq C_{\varepsilon}|d|^{-1 / 2+3 \sigma+\varepsilon}\left\|\chi_{2 d} R\left(E+i 0 ; H_{d}\right) g_{1 d}\right\|+C_{N}|d|^{-N}
\end{aligned}
$$

for any $\varepsilon, 0<\varepsilon \ll 1$, and $N \gg 1$.

We shall show (5.13). By the resolvent identity,

$$
R\left(E+i 0 ; H_{d}\right)=R\left(E+i 0 ; K_{1 d}\right)-R\left(E+i 0 ; H_{d}\right) W_{2 d} R\left(E+i 0 ; K_{1 d}\right) .
$$

We consider the second operator on the right side and we evaluate

$$
Q=\chi_{2 d} R\left(E+i 0 ; H_{d}\right) W_{2 d} R\left(E+i 0 ; K_{1 d}\right) \chi_{1 d}
$$


To do this, we decompose $W_{2 d}$ as in (5.7), and we set

$$
\begin{aligned}
Q_{0} & =\chi_{2 d} R\left(E+i 0 ; H_{d}\right) g_{2 d}^{2} W_{2 d} R\left(E+i 0 ; K_{1 d}\right) \chi_{1 d} \\
Q_{\infty} & =\chi_{2 d} R\left(E+i 0 ; H_{d}\right) W_{\infty} R\left(E+i 0 ; K_{1 d}\right) \chi_{1 d} \\
Q_{ \pm} & =\chi_{2 d} R\left(E+i 0 ; H_{d}\right) W_{ \pm} R\left(E+i 0 ; K_{1 d}\right) \chi_{1 d}
\end{aligned}
$$

Lemma 5.3 remains true for the pair $\left(b_{1 d}, b_{2 d}\right)=\left(\chi_{1 d}, g_{2 d}\right)$. Hence we have

$$
\left\|g_{2 d} W_{2 d} R\left(E+i 0 ; K_{1 d}\right) \chi_{1 d}\right\| \simeq O\left(|d|^{-1 / 2+3 \sigma}\right)
$$

by elliptic estimate. This implies that

$$
\left\|Q_{0}\right\| \leq C_{\varepsilon}|d|^{-1 / 2+3 \sigma+\varepsilon}\left\|\chi_{2 d} R\left(E+i 0 ; H_{d}\right) g_{2 d}\right\|
$$

The operator $W_{-} R\left(E+i 0 ; K_{1 d}\right)$ is approximated by (5.8). Since the free particle reaching $\operatorname{supp} W_{-}$at $t=0$ does not pass over $\operatorname{supp} \chi_{1 d}$ for $t<0$, we have

$$
W_{-} R\left(E+i 0 ; K_{1 d}\right) \chi_{1 d}=\tilde{r}_{N}+\tilde{r}_{N} R\left(E+i 0 ; K_{1 d}\right) \chi_{1 d}
$$

and hence $\left\|Q_{-}\right\|=O\left(|d|^{-N}\right)$ for any $N \gg 1$ by Lemma 3.1. A similar argument applies to $Q_{+}$. The operator $R\left(E+i 0 ; H_{d}\right) W_{+}$is approximated by (5.12). If $M \gg 1$, then the free particle starting from $\operatorname{supp} W_{+}$at $t=0$ does not pass over supp $\chi_{2 d}$ for $t>0$, so that

$$
\chi_{2 d} R\left(E+i 0 ; H_{d}\right) W_{+}=\tilde{r}_{N}+\chi_{2 d} R\left(E+i 0 ; H_{d}\right) \tilde{r}_{N} .
$$

This shows that $\left\|Q_{+}\right\|=O\left(|d|^{-N}\right)$ by Lemma 3.1. The approximation for $W_{\infty} R\left(E+i 0 ; K_{1 d}\right) \chi_{1 d}$ takes the form

$$
W_{\infty} R\left(E+i 0 ; K_{1 d}\right) \chi_{1 d}=\tilde{r}_{N}+\tilde{r}_{N} R\left(E+i 0 ; K_{1 d}\right) \chi_{1 d}
$$

as in the proof of Lemma 5.3. Hence we obtain $\left\|Q_{\infty}\right\|=O\left(|d|^{-N}\right)$ by Lemma 3.1 again. Thus we combine all the estimates above to get

$$
\|Q\| \leq C_{\varepsilon}|d|^{-1 / 2+3 \sigma+\varepsilon}\left\|\chi_{2 d} R\left(E+i 0 ; H_{d}\right) g_{2 d}\right\|+C_{N}|d|^{-N}
$$

and (5.13) follows from Lemma 5.3. A similar argument applies to

$$
\begin{aligned}
& \chi_{1 d} R\left(E+i 0 ; H_{d}\right) W_{2 d} R\left(E+i 0 ; K_{1 d}\right) \chi_{1 d}, \\
& \chi_{2 d} R\left(E+i 0 ; H_{d}\right) W_{1 d} R\left(E+i 0 ; K_{2 d}\right) \chi_{2 d},
\end{aligned}
$$

and (5.14) and (5.15) are obtained. 
Proposition 5.2 is still valid for $\left(b_{1 d}, b_{2 d}\right)=\left(g_{1 d}, \chi_{2 d}\right)$ or $\left(\chi_{1 d}, g_{2 d}\right)$. In fact,

$$
\operatorname{supp} g_{1 d}=\left\{x:|x|<2 M|d|^{\sigma}\right\} \subset\left\{x:|x|<C|d|^{\sigma+\varepsilon}\right\}, \quad \varepsilon>0,
$$

for $|d| \gg 1$, so that the proposition is true with $\sigma$ replaced by $\sigma+\varepsilon$. Thus we have

$$
\left\|\chi_{2 d} R\left(E+i 0 ; H_{d}\right) g_{1 d}\right\|+\left\|\chi_{1 d} R\left(E+i 0 ; H_{d}\right) g_{2 d}\right\| \simeq O\left(|d|^{3 \sigma}\right) .
$$

The bounds in the lemma are derived by combining this estimate with the three inequalities (5.13) through (5.15). Since $\left\|\chi_{j d} R\left(E+i 0 ; K_{j d}\right) \chi_{j d}\right\| \simeq O\left(|d|^{\sigma}\right)$ by the principle of limiting absorption, it follows from (5.14), (5.15) and (5.16) that

$$
\left\|\chi_{j d} R\left(E+i 0 ; H_{d}\right) \chi_{j d}\right\| \simeq O\left(|d|^{\sigma}\right) .
$$

This remains true for $g_{j d}$ for the same reason as above. We combine (5.13) and (5.17) to obtain that

$$
\left\|\chi_{2 d} R\left(E+i 0 ; H_{d}\right) \chi_{1 d}\right\| \simeq O\left(|d|^{-1 / 2+4 \sigma}\right),
$$

which is also valid for $g_{j d}$. Thus it follows again from (5.14) and (5.15) that

$$
\left\|\chi_{j d}\left(R\left(E+i 0 ; H_{d}\right)-R\left(E+i 0 ; K_{j d}\right)\right) \chi_{j d}\right\| \simeq O\left(|d|^{-1+7 \sigma}\right) .
$$

Recall that $R\left(E+i 0 ; K_{1 d}\right)=p_{2 d} R\left(E+i 0 ; H\left(A_{1}\right)\right) q_{2 d}$ with $q_{2 d}=1 / p_{2 d}$. By (4.8), $p_{2 d}$ behaves like

$$
p_{2 d}(x)=e^{i \alpha_{2} \gamma(x-d ; \hat{d})}=e^{i \alpha_{2} \gamma(-d ; \hat{d})}+O\left(|d|^{-1+\sigma}\right)=e^{i \alpha_{2} \pi}+O\left(|d|^{-1+\sigma}\right)
$$

on $\operatorname{supp} \chi_{1 d}$. Thus we have

$$
\left\|\chi_{1 d}\left(R\left(E+i 0 ; H_{d}\right)-R\left(E+i 0 ; H\left(A_{1}\right)\right)\right) \chi_{1 d}\right\| \simeq O\left(|d|^{-1+7 \sigma}\right) .
$$

A similar argument applies to $\chi_{2 d} R\left(E+i 0 ; H_{d}\right) \chi_{2 d}$, and the proof of the lemma is complete.

\section{§6. Proof of Propositions 5.1 and 5.2}

We here prove Propositions 5.1 and 5.2. Throughout the section, we fix $\sigma_{1}$ as $\sigma \leq \sigma_{1} \ll 1$ and take $\rho$ as

$$
1 / 2<\rho<\sigma_{1} / 4+1 / 2
$$


On the other hand, $\sigma_{2}$ is assumed to satisfy

$$
0<\sigma_{2}<\left(\sigma_{1} / 4-(\rho-1 / 2)\right) / 3
$$

for $\rho>1 / 2$ as above. We further write $h_{2 d}(x)$ for the characteristic function of $\left\{|x-d|<c|d|^{\kappa}\right\}$ with $0<\kappa \ll 1$ small enough, $c>0$ being fixed arbitrarily.

6.1. The argument here is based on the following proposition.

Proposition 6.1. $\quad$ Assume that $\rho$ fulfills (6.1). Then

$$
\left\|\langle x\rangle^{\rho} W_{1 d} R\left(E+i 0 ; K_{0 d}\right) h_{2 d}\right\|=O\left(|d|^{-\nu}\right)
$$

with $\nu=\sigma_{1} / 4-(\rho-1 / 2)-\kappa$.

By (4.10), $W_{1 d}$ takes the form $W_{1 d}=2 i e_{1 d} \cdot \nabla+e_{0 d}$, where

$$
e_{1 d}(x)=\left(\alpha_{1}-\zeta_{1 d}^{\prime}(\gamma)\right) \nabla \gamma=O\left(|d|^{\sigma_{1} / 2}\right) \nabla \gamma, \quad \gamma=\gamma(x ;-\hat{d}),
$$

and $e_{0 d}(x)=O\left(|d|^{\sigma_{1}}\right)|x|^{-2}$ in $\left\{|x|>|d|^{\sigma_{1}}\right\}$. The proof of the proposition depends on this special form.

Lemma 6.1. Recall that $\pi_{1 d}$ is the characteristic function of $\Pi_{1 d}$. Then

$$
\left\|\langle x\rangle^{\rho-2} \pi_{1 d} R\left(E+i 0 ; K_{0 d}\right) h_{2 d}\right\|=O\left(|d|^{-\left(\sigma_{1}+\nu\right)}\right)
$$

with $\nu=1 / 2-\sigma_{1}-\kappa>0$.

Proof. Let $D_{1}=\left\{x \in \Pi_{1 d}, \quad y \in \operatorname{supp} h_{2 d}\right\}$. We evaluate the integral

$$
I=\iint_{D_{1}}\langle x\rangle^{2(\rho-2)}\left|G_{d}(x, y ; E)\right|^{2} d y d x
$$

where $G_{d}(x, y ; E)$ is the kernel of $R\left(E+i 0 ; K_{0 d}\right)$. Since $|x-y|>c(|x|+|d|)$ for $(x, y) \in D_{1}$, it follows from (5.1) that

$$
\begin{aligned}
I & =O\left(|d|^{2 \kappa}\right) \int_{\Pi_{1 d}}\langle x\rangle^{2(\rho-2)}(|x|+|d|)^{-1} d x \\
& =O\left(|d|^{2 \kappa}\right) O\left(|d|^{-1}\right) \int_{0}^{\infty}(1+r)^{2(\rho-2)} r d r=O\left(|d|^{-2(1 / 2-\kappa)}\right) .
\end{aligned}
$$

Hence we have $I=O\left(|d|^{-2\left(\sigma_{1}+\nu\right)}\right)$ with $\nu$ in the lemma. This proves the lemma. 
Lemma 6.2. If $g(x)$ is bounded with support in $\left\{x \in \Pi_{1 d}:|x|>\right.$ $\left.C|d|^{\sigma_{1}}\right\}$ for $C \gg 1$ as in (4.1), then

$$
\left\|\langle x\rangle^{\rho} g(\nabla \gamma \cdot \nabla) R\left(E+i 0 ; K_{0 d}\right) h_{2 d}\right\|=O\left(|d|^{-\left(\sigma_{1} / 2+\nu\right)}\right)
$$

with $\nu=\sigma_{1} / 4-(\rho-1 / 2)-\kappa$, where $\gamma=\gamma(x ;-\hat{d})$.

Proof. Let $D_{2}=\left\{x \in \Pi_{1 d}, \quad|x|>C|d|^{\sigma_{1}}, \quad y \in \operatorname{supp} h_{2 d}\right\}$. We calculate

$$
I(x, y)=(\nabla \gamma \cdot \nabla) \exp (i \sqrt{E}|x-y|)
$$

for $(x, y) \in D_{2}$. A direct calculation yields

$$
I(x, y)=i \sqrt{E}|x|^{-1}|x-y|^{-1}|y|\left(\hat{x}_{2} \hat{y}_{1}-\hat{x}_{1} \hat{y}_{2}\right) \exp (i \sqrt{E}|x-y|),
$$

where $\hat{x}=\left(\hat{x}_{1}, \hat{x}_{2}\right)$. If $(x, y) \in D_{2}$, then $\hat{x}=-\hat{d}+O\left(|d|^{-\sigma_{1} / 2}\right)$ and $\hat{y}=$ $\hat{d}+O\left(|d|^{-1+\kappa}\right)$, so that

$$
\hat{x}_{2} \hat{y}_{1}-\hat{x}_{1} \hat{y}_{2}=O\left(|d|^{-\sigma_{1} / 2}\right) .
$$

Thus we have

$$
I(x, y)=O\left(|d|^{1-\sigma_{1} / 2}\right)|x|^{-1}|x-y|^{-1}
$$

uniformly in $(x, y) \in D_{2}$. Note that $|\hat{x}+\hat{d}|<|d|^{-\sigma_{1} / 2}$ when $x \in \Pi_{1 d}$ satisfies $|x|>C|d|^{\sigma_{1}}$. Then the integral $I$ below is evaluated as

$$
\begin{aligned}
I & =\iint_{D_{2}}|x|^{2 \rho}|I(x, y)|^{2}|x-y|^{-1} d y d x \\
& =O\left(|d|^{2-\sigma_{1}+2 \kappa}\right) O\left(|d|^{-\sigma_{1} / 2}\right) \int_{0}^{\infty} r^{2 \rho-1}(r+|d|)^{-3} d r=O\left(|d|^{-\left(\sigma_{1}+2 \nu\right)}\right)
\end{aligned}
$$

for $\nu$ as in the lemma. The lemma follows from this estimate.

Proof of Proposition 6.1. Let $h_{1 d}(x)$ be bounded with support in $\{|x|<$ $\left.C|d|^{\sigma_{1}}\right\}$. Then it follows from (5.1) that

$$
\left\|\langle x\rangle^{\rho} h_{1 d} R\left(E+i 0 ; K_{0 d}\right) h_{2 d}\right\|=O\left(|d|^{-\mu}\right)
$$

with $\mu=1 / 2-(\rho+1) \sigma_{1}-\kappa>0$. Hence

$$
\left\|\langle x\rangle^{\rho} h_{1 d} W_{1 d} R\left(E+i 0 ; K_{0 d}\right) h_{2 d}\right\|=O\left(|d|^{-\mu}\right)
$$

by elliptic estimate. Since $\mu>\nu=\sigma_{1} / 4-(\rho-1 / 2)-\kappa$ for $\sigma_{1}$ small enough, the proposition is obtained from Lemmas 6.1 and 6.2. 
Lemma 6.3. One has

$$
\left\|h_{2 d} R\left(E+i 0 ; K_{1 d}\right) \pi_{1 d}\langle x\rangle^{-\rho}\right\|=O\left(|d|^{-\nu}\right)
$$

with $\nu=\sigma_{1} / 4-(\rho-1 / 2)-\kappa$.

Proof. By the resolvent identity, we have

$$
R\left(E+i 0 ; K_{1 d}\right)=R\left(E+i 0 ; K_{0 d}\right)-R\left(E+i 0 ; K_{0 d}\right) W_{1 d} R\left(E+i 0 ; K_{1 d}\right) .
$$

We can show

$$
\left\|h_{2 d} R\left(E+i 0 ; K_{0 d}\right) \pi_{1 d}\langle x\rangle^{-\rho}\right\|=O\left(|d|^{-\left(\sigma_{1} / 4+(\rho-1 / 2)-\kappa\right)}\right),
$$

which follows from (5.1) by evaluating the Hilbert-Schmidt norm. If we decompose $W_{1 d}$ into $W_{1 d}=\left(W_{1 d}\langle x\rangle^{\rho}\right)\langle x\rangle^{-\rho}$ for the second operator, then the lemma is obtained from Proposition 6.1.

Lemma 6.4. Assume that $\kappa=\sigma_{2}$ for $\sigma_{2}$ as in (6.2). Then

$$
\left\|\langle x\rangle^{\rho} W_{1 d} R\left(E+i 0 ; K_{2 d}\right) h_{2 d}\right\| \simeq O\left(|d|^{-\nu}\right)
$$

with $\nu=\sigma_{1} / 4-(\rho-1 / 2)-2 \sigma_{2}>0$.

Proof. By the resolvent identity, we have

$$
R\left(E+i 0 ; K_{2 d}\right)=R\left(E+i 0 ; K_{0 d}\right)-R\left(E+i 0 ; K_{0 d}\right) W_{2 d} R\left(E+i 0 ; K_{2 d}\right) .
$$

By Proposition 6.1, the first operator on the right side is estimated as

$$
\left\|\langle x\rangle^{\rho} W_{1 d} R\left(E+i 0 ; K_{0 d}\right) h_{2 d}\right\|=O\left(|d|^{-\mu}\right)
$$

with $\mu=\sigma_{1} / 4-(\rho-1 / 2)-\sigma_{2}$. We estimate the second operator. To do this, we decompose $W_{2 d}$ into the sum of four operators

$$
W_{2 d}=g_{2 d}^{2} W_{2 d}+W_{\infty}\left(x, D_{x}\right)+W_{-}\left(x, D_{x}\right)+W_{+}\left(x, D_{x}\right)
$$

as in (5.7), where $g_{2 d}(x)=\chi\left(|x-d| / M|d|^{\sigma_{2}}\right)$ for $M \gg 1$. By the principle of limiting absorption,

$$
\langle x-d\rangle^{-\rho} R\left(E+i 0 ; K_{2 d}\right)\langle x-d\rangle^{-\rho}: L^{2} \rightarrow L^{2}
$$


is bounded uniformly in $d$, and hence we have

$$
\left\|g_{2 d} W_{2 d} R\left(E+i 0 ; K_{2 d}\right) h_{2 d}\right\| \simeq O\left(|d|^{\sigma_{2}}\right)
$$

by elliptic estimate. Thus it follows from Proposition 6.1 that

$$
\left\|\langle x\rangle^{\rho} W_{1 d} R\left(E+i 0 ; K_{0 d}\right) g_{2 d}^{2} W_{2 d} R\left(E+i 0 ; K_{2 d}\right) h_{2 d}\right\| \simeq O\left(|d|^{-\nu}\right)
$$

for $\nu$ as in the lemma. The other operators with $W_{\infty}\left(x, D_{x}\right)$ and $W_{ \pm}\left(x, D_{x}\right)$ are evaluated in almost the same way as in the proof of Lemmas 5.3 and 5.4. These operators be shown to obey the bound $O\left(|d|^{-N}\right)$ for any $N \gg 1$. This proves the lemma.

Lemma 6.5. Let $g_{2 d}(x)=\chi\left(|x-d| / M|d|^{\sigma_{2}}\right)$ and let $W_{+}=\left(1-g_{2 d}^{2}\right)$ $W_{2 d} \beta_{+}$be as in (5.7). Then

$$
\left\|\langle x\rangle^{\rho} W_{1 d} R\left(E+i 0 ; K_{2 d}\right) W_{+}\langle x\rangle^{\rho}\right\|=O\left(|d|^{-N}\right)
$$

for any $N \gg 1$.

Proof. We give only a sketch for a proof. The symbol $W_{+}(x, \xi)$ has support in an outgoing region. The idea is to construct an approximation for $R\left(E+i 0 ; K_{2 d}\right) W_{+}$. This is constructed in the same way as $R\left(E+i 0 ; H_{d}\right) W_{+}$ (see $(5.12))$, and it takes the form

$$
j_{-d} e^{i \alpha_{2} \gamma(x-d ;-\hat{d})} p_{1 d} R\left(E+i 0 ; H_{0}\right) \tilde{\beta}_{+} q_{1 d} e^{-i \alpha_{2} \gamma(x-d ;-\hat{d})} W_{+}
$$

with $R\left(E+i 0 ; K_{2 d}\right) \tilde{r}_{N}$ as an error operator. If we take account of the fact that the free particle starting from supp $W_{+}$at time $t=0$ does not pass over $\Pi_{1 d}$ for $t>0$, then we have

$$
\langle x\rangle^{\rho} W_{1 d} R\left(E+i 0 ; K_{2 d}\right) W_{+}\langle x\rangle^{\rho}=\tilde{r}_{N}+\langle x\rangle^{\rho} W_{1 d} R\left(E+i 0 ; K_{2 d}\right) \tilde{r}_{N} .
$$

Hence the lemma follows from Lemma 5.4.

Lemma 6.6. Let $W_{+}$be as above. If $L \gg 1$, then

$$
\left\|r_{L} R\left(E+i 0 ; K_{2 d}\right) W_{+}\langle x\rangle^{\rho}\right\|=O\left(|d|^{-2 L / 3}\right) .
$$

Proof. The operator $R\left(E+i 0 ; K_{2 d}\right) W_{+}$is approximated by (6.3). Hence the lemma follows from Lemma 2.6. 
6.2. We are now in a position to prove the two propositions in question.

Proof of Proposition 5.1. Throughout the proof, $\sigma_{1}$ is fixed as $\sigma_{1}=\sigma$, and $\sigma_{2}$ is specified by (6.2) with $\sigma_{1}=\sigma$. We consider only the first operator on the left side, and we write

$$
X=r_{L} R\left(E+i 0 ; H_{d}\right) \pi_{1 d}\langle x\rangle^{-\rho}
$$

for this operator. Then

$X=r_{L} R\left(E+i 0 ; K_{1 d}\right) \pi_{1 d}\langle x\rangle^{-\rho}-r_{L} R\left(E+i 0 ; H_{d}\right) W_{2 d} R\left(E+i 0 ; K_{1 d}\right) \pi_{1 d}\langle x\rangle^{-\rho}$

by the resolvent identity. It is easy to see that the first operator satisfies

$$
\left\|r_{L} R\left(E+i 0 ; K_{1 d}\right) \pi_{1 d}\langle x\rangle^{-\rho}\right\|=O\left(|d|^{-2 L / 3}\right) .
$$

To estimate the second operator, we decompose $W_{2 d}$ into the sum of four operators

$$
W_{2 d}=g_{2 d}^{2} W_{2 d}+W_{\infty}\left(x, D_{x}\right)+W_{+}\left(x, D_{x}\right)+W_{-}\left(x, D_{x}\right)
$$

as in (5.7), where $g_{2 d}(x)=\chi\left(|x-d| / M|d|^{\sigma_{2}}\right)$ for $M \gg 1$. According to the above decomposition, we set

$$
\begin{aligned}
X_{0} & =r_{L} R\left(E+i 0 ; H_{d}\right) g_{2 d}^{2} W_{2 d} R\left(E+i 0 ; K_{1 d}\right) \pi_{1 d}\langle x\rangle^{-\rho} \\
X_{\infty} & =r_{L} R\left(E+i 0 ; H_{d}\right) W_{\infty} R\left(E+i 0 ; K_{1 d}\right) \pi_{1 d}\langle x\rangle^{-\rho} \\
X_{ \pm} & =r_{L} R\left(E+i 0 ; H_{d}\right) W_{ \pm} R\left(E+i 0 ; K_{1 d}\right) \pi_{1 d}\langle x\rangle^{-\rho}
\end{aligned}
$$

Then we have

$$
\|X\| \leq C_{L}|d|^{-L / 2}+\left\|X_{0}\right\|+\left\|X_{\infty}\right\|+\left\|X_{-}\right\|+\left\|X_{+}\right\|
$$

We apply the same argument as in the proof of Lemmas 5.3 or 5.4 to evaluate $X_{-}$and $X_{\infty}$. The operator $W_{-} R\left(E+i 0 ; K_{1 d}\right)$ is approximated by (5.8). The free particle reaching supp $W_{-}$at $t=0$ does not pass over $\Pi_{1 d}$ for $t<0$. Hence we have

$$
W_{-} R\left(E+i 0 ; K_{1 d}\right) \pi_{1 d}\langle x\rangle^{-\rho}=\tilde{r}_{N}+\tilde{r}_{N} R\left(E+i 0 ; K_{1 d}\right) \pi_{1 d}\langle x\rangle^{-\rho} .
$$

This shows that

$$
\left\|X_{-}\right\|=O\left(|d|^{-N}\right)\left\|r_{L} R\left(E+i 0 ; H_{d}\right) r_{L}\right\| .
$$


The symbol $K_{1 d}(x, \xi)$ is invertible on supp $W_{\infty}$. Since $\Pi_{1 d}$ and $\operatorname{supp} W_{\infty}$ do not intersect with each other, it follows that

$$
W_{\infty} R\left(E+i 0 ; K_{1 d}\right) \pi_{1 d}\langle x\rangle^{-\rho}=\tilde{r}_{N}+\tilde{r}_{N} R\left(E+i 0 ; K_{1 d}\right) \pi_{1 d}\langle x\rangle^{-\rho} .
$$

This also implies that

$$
\left\|X_{\infty}\right\|=O\left(|d|^{-N}\right)\left\|r_{L} R\left(E+i 0 ; H_{d}\right) r_{L}\right\| .
$$

We evaluate $r_{L} R\left(E+i 0 ; H_{d}\right) r_{L}$. This is represented as

$$
r_{L} R\left(E+i 0 ; K_{2 d}\right) r_{L}-r_{L} R\left(E+i 0 ; H_{d}\right) W_{1 d} R\left(E+i 0 ; K_{2 d}\right) r_{L} .
$$

If we decompose $W_{1 d}$ into $W_{1 d}=\left(\pi_{1 d}\langle x\rangle^{-\rho}\right)\left(\langle x\rangle^{\rho} W_{1 d}\right)$, then Lemma 5.4 shows that

$$
\left\|r_{L} R\left(E+i 0 ; H_{d}\right) r_{L}\right\|=O\left(|d|^{-L}\right)+O\left(|d|^{-L / 2}\right)\|X\|
$$

and hence we obtain

$$
\left\|X_{\infty}\right\|+\left\|X_{-}\right\| \leq C_{N}\left(|d|^{-N}+|d|^{-N}\|X\|\right)
$$

for any $N \gg 1$. We consider $X_{+}$. We decompose it into

$$
X_{+}=\left(r_{L} R\left(E+i 0 ; H_{d}\right) W_{+}\langle x\rangle^{\rho}\right)\left(\langle x\rangle^{-\rho} R\left(E+i 0 ; K_{1 d}\right) \pi_{1 d}\langle x\rangle^{-\rho}\right) .
$$

The second operator is bounded uniformly in $d$, and the first one is rewritten as

$$
r_{L} R\left(E+i 0 ; K_{2 d}\right) W_{+}\langle x\rangle^{\rho}-r_{L} R\left(E+i 0 ; H_{d}\right) W_{1 d} R\left(E+i 0 ; K_{2 d}\right) W_{+}\langle x\rangle^{\rho} .
$$

By Lemmas 6.5 and 6.6, we have

$$
\left\|r_{L} R\left(E+i 0 ; H_{d}\right) W_{+}\langle x\rangle^{\rho}\right\|=O\left(|d|^{-2 L / 3}\right)+O\left(|d|^{-N}\right)\|X\| .
$$

Thus $X$ satisfies

$$
\|X\| \leq C_{L N}\left(|d|^{-2 L / 3}+|d|^{-N}\|X\|\right)+\left\|X_{0}\right\| .
$$

We estimate $X_{0}$. By elliptic estimate, it follows from Lemma 6.3 with $\kappa=\sigma_{2}$ that

$$
\left\|g_{2 d} W_{2 d} R\left(E+i 0 ; K_{1 d}\right) \pi_{1 d}\langle x\rangle^{-\rho}\right\|=O\left(|d|^{-\nu}\right)
$$

for $\nu=\sigma_{1} / 4-(\rho-1 / 2)-\sigma_{2}>0$. Hence $X_{0}$ obeys the bound

$$
\left\|X_{0}\right\|=o(1)\left\|r_{L} R\left(E+i 0 ; H_{d}\right) g_{2 d}\right\|
$$


We further write $r_{L} R\left(E+i 0 ; H_{d}\right) g_{2 d}$ as

$$
r_{L} R\left(E+i 0 ; K_{2 d}\right) g_{2 d}-r_{L} R\left(E+i 0 ; H_{d}\right) W_{1 d} R\left(E+i 0 ; K_{2 d}\right) g_{2 d}
$$

Then we obtain

$$
\left\|X_{0}\right\|=O\left(|d|^{-2 L / 3}\right)+o(1)\|X\|
$$

by Lemma 6.4. This, together with (6.4), yields the desired bound and the proof is complete.

Proof of Proposition 5.2. Throughout the proof, $\sigma_{2}$ is fixed as $\sigma_{2}=\sigma$, and $\sigma_{1}$ and $\rho$ are chosen to fulfill (6.1) and (6.2). The proof is done by modifying slightly the argument in the proof of Proposition 5.1. It suffices to show that

$$
\|Y\|=\left\|b_{2 d} R\left(E+i 0 ; H_{d}\right) \pi_{1 d}\langle x\rangle^{-\rho}\right\|=O\left(|d|^{2 \sigma}\right),
$$

because supp $b_{1 d} \subset \Pi_{1 d}$ for $\sigma_{1}>\sigma$. By the resolvent identity, we have $Y=b_{2 d} R\left(E+i 0 ; K_{1 d}\right) \pi_{1 d}\langle x\rangle^{-\rho}-b_{2 d} R\left(E+i 0 ; H_{d}\right) W_{2 d} R\left(E+i 0 ; K_{1 d}\right) \pi_{1 d}\langle x\rangle^{-\rho}$.

The first operator satisfies

$$
\left\|b_{2 d} R\left(E+i 0 ; K_{1 d}\right) \pi_{1 d}\langle x\rangle^{-\rho}\right\|=o(1)
$$

by Lemma 6.3. We set

$$
\begin{aligned}
Y_{0} & =b_{2 d} R\left(E+i 0 ; H_{d}\right) g_{2 d}^{2} W_{2 d} R\left(E+i 0 ; K_{1 d}\right) \pi_{1 d}\langle x\rangle^{-\rho}, \\
Y_{\infty} & =b_{2 d} R\left(E+i 0 ; H_{d}\right) W_{\infty} R\left(E+i 0 ; K_{1 d}\right) \pi_{1 d}\langle x\rangle^{-\rho}, \\
Y_{ \pm} & =b_{2 d} R\left(E+i 0 ; H_{d}\right) W_{ \pm} R\left(E+i 0 ; K_{1 d}\right) \pi_{1 d}\langle x\rangle^{-\rho},
\end{aligned}
$$

where $g_{2 d}(x)=\chi\left(|x-d| / M|d|^{\sigma}\right)$ for $M \gg 1$. To evaluate $Y_{-}$and $Y_{\infty}$, we construct approximations for $W_{-} R\left(E+i 0 ; K_{1 d}\right)$ and $W_{\infty} R\left(E+i 0 ; K_{1 d}\right)$ as in the proof of Proposition 5.1. Since $\operatorname{supp} b_{2 d} \subset \Pi_{2 d}$, we have

$$
\left\|b_{2 d} R\left(E+i 0 ; H_{d}\right) r_{N}\right\|=O\left(|d|^{-N / 2}\right)
$$

by Proposition 5.1. Hence it follows that $\left\|Y_{-}\right\|+\left\|Y_{\infty}\right\|=O\left(|d|^{-N}\right)$ for any $N \gg 1$. To evaluate $Y_{+}$, we construct the approximation for $R\left(E+i 0 ; H_{d}\right) W_{+}$ by (5.12). If $M \gg 1$, then the free particle starting from $\operatorname{supp} W_{+}$at $t=0$ does not pass over $\operatorname{supp} b_{2 d}$ for $t>0$. Hence we have $\left\|Y_{+}\right\|=O\left(|d|^{-N}\right)$ by Proposition 5.1 again. Thus it follows that $\|Y\|=o(1)+\left\|Y_{0}\right\|$. The operator 
$Y_{0}$ is also evaluated in almost the same way as $X_{0}$. We have chosen $\sigma_{1}$ and $\rho$ to fulfill (6.1) and (6.2) for $\sigma_{2}=\sigma$. By Lemma 6.3, we have

$$
\left\|Y_{0}\right\|=o(1)\left\|b_{2 d} R\left(E+i 0 ; H_{d}\right) g_{2 d}\right\|
$$

while $b_{2 d} R\left(E+i 0 ; H_{d}\right) g_{2 d}$ obeys

$$
\left\|b_{2 d} R\left(E+i 0 ; H_{d}\right) g_{2 d}\right\|=\left\|b_{2 d} R\left(E+i 0 ; K_{2 d}\right) g_{2 d}\right\|+o(1)\|Y\|
$$

by Lemma 6.4 . Hence

$$
\left\|Y_{0}\right\|=O\left(|d|^{2 \sigma}\right)+o(1)\|Y\| .
$$

This completes the proof.

\section{$\S 7 . \quad$ Proof of Theorem 1.2 : Integer Flux Case}

The aim here is to prove Theorem 1.2. We assume for brevity that the flux $\alpha_{2}$ is an integer.

7.1. The representation for amplitudes in Lemma 2.4 does not work for the case $\omega= \pm \hat{d}$ or $\tilde{\omega}= \pm \hat{d}$. We represent the scattering amplitude in a different form to prove the theorem. Let $b \in C_{0}^{\infty}\left(\boldsymbol{R}^{2}\right)$ be given magnetic field with flux $\alpha$. Assume that

$$
\operatorname{supp} b \subset\{|x|<M\}
$$

for some $M>0$. By Lemma 2.1, we can construct a magnetic potential $A(x)$ associated with $b$ such that

$$
A(x)=A_{\alpha}(x)=\alpha\left(-x_{2} /|x|^{2}, x_{1} /|x|^{2}\right)
$$

for $|x|>2 M$. We introduce the auxiliary Hamiltonian

$$
H_{\alpha}=H\left(A_{\alpha}\right)=\left(-i \nabla-A_{\alpha}\right)^{2} .
$$

This Hamiltonian has the $\delta$-like magnetic field $\nabla \times A_{\alpha}=2 \pi \alpha \delta(x)$ at the origin, and it admits a self-adjoint realization with domain

$$
\mathcal{D}\left(H_{\alpha}\right)=\left\{u \in L^{2}\left(\boldsymbol{R}^{2}\right): H_{\alpha} u \in L^{2}\left(\boldsymbol{R}^{2}\right), \lim _{|x| \rightarrow 0}|u(x)|<\infty\right\},
$$

where $H_{\alpha} u$ is understood in the distributional sense. We denote by the same notation $H_{\alpha}$ this self-adjoint extension. The operator $H_{\alpha}$ has the polar coordinate decomposition

$$
H_{\alpha} \simeq \sum_{l \in Z} \oplus\left(-\partial_{r}^{2}+\left(\nu^{2}-1 / 4\right) r^{-2}\right), \quad \nu=|l-\alpha|,
$$


and the generalized eigenfunction $\varphi_{\mp}(x ; \omega, E), H_{\alpha} \varphi_{\mp}=E \varphi_{\mp}$, is given by

$$
\varphi_{\mp}(x ; \omega, E)=\sum_{l \in Z} \exp ( \pm i \nu \pi / 2) \exp (i l \gamma(x ; \pm \omega)) J_{\nu}(\sqrt{E}|x|),
$$

where the series is convergent locally uniformly. We refer to [2], [12] for the details of the spectral properties of $H_{\alpha}$. By (7.2), the difference $H(A)-H_{\alpha}$ is a perturbation of short-range class. The scattering amplitude $f(\omega \rightarrow \tilde{\omega} ; E)$ for the pair $\left(H(A), H_{0}\right)$ is obtained as the sum of two amplitudes for $\left(H_{\alpha}, H_{0}\right)$ and $\left(H(A), H_{\alpha}\right)$, and the amplitude for $\left(H(A), H_{\alpha}\right)$ is represented in terms of the eigenfunction $\varphi_{\mp}(x ; \omega, E)$ of $H_{\alpha}$.

To formulate the representation formula for $f(\omega \rightarrow \tilde{\omega} ; E)$, we fix the new notation. Let $M>0$ be as in (7.1). We set $\chi_{\infty M}(x)=1-\chi_{M}(x)$, where $\chi_{M}(x)=\chi(|x| / 2 M)$ and $\chi \in C_{0}^{\infty}[0, \infty)$ is the cut-off function with property (2.3). Note that $H(A)=H_{\alpha}$ on $\operatorname{supp} \chi_{\infty}$. We define $\psi_{+}(x ; \omega, E)$ as

$$
\psi_{+}(x ; \omega, E)=\left(\chi_{\infty M}-R(E+i 0 ; H(A)) D_{M}\right) \varphi_{+}(x ; \omega, E),
$$

where

$$
D_{M}=\left[H(A), \chi_{\infty M}\right]=H(A) \chi_{\infty M}-\chi_{\infty M} H(A) .
$$

As is easily seen, $\psi_{+}(x ; \omega, E)$ is a unique solution to equation $(H(A)-E) \psi_{+}=$ 0 such that $\psi_{+}-\varphi_{+}(x ; \omega, E)$ satisfies the outgoing radiation condition at infinity. We have derived the following representation formula in [14].

Proposition 7.1. Let the notation be as above. Assume that $\omega \neq \omega^{\prime}$. Then the scattering amplitude $f(\omega \rightarrow \tilde{\omega} ; E)$ for $\left(H(A), H_{0}\right)$ is represented as

$$
f(\omega \rightarrow \tilde{\omega} ; E)=c(E)\left(f_{\alpha}(\tilde{\omega}-\omega)-(i / 4 \pi) g_{\alpha}(\omega \rightarrow \tilde{\omega} ; E)\right)
$$

with $c(E)=(2 \pi / i \sqrt{E})^{1 / 2}$, where

$$
f_{\alpha}(\theta)=-(i \sin \alpha \pi / \pi) \exp (i[\alpha] \theta) F_{0}(\theta)
$$

with $F_{0}(\theta)=e^{i \theta} /\left(e^{i \theta}-1\right), \theta \in S^{1}$ being identified with the azimuth angle from the positive $x_{1}$ axis, and

$$
g_{\alpha}(\omega \rightarrow \tilde{\omega} ; E)=\left(\psi_{+}(\omega, E), D_{M} \varphi_{-}(\tilde{\omega}, E)\right)
$$

with $\psi_{+}(\omega, E)=\psi_{+}(x ; \omega, E)$ defined by $(7.4)$. 
Remark 7.1. The first term $c(E) f_{\alpha}(\tilde{\omega}-\omega)$ describes the scattering amplitude for $\left(H_{\alpha}, H_{0}\right)$ (see [2], [3], [12]).

7.2. Another important ingredient to prove Theorem 1.2 is the asymptotic behavior as $|x| \rightarrow \infty$ of the eigenfunction $\varphi_{\mp}(x ; \omega, E)$ of $H_{\alpha}$. The result has been already known by [2], [3], [10].

Proposition 7.2. Let $\varphi_{\mp}(x ; \omega, E)$ be defined by (7.3). Then one has the following statements.

(1) If $x /|x| \neq \omega$, then $\varphi_{+}(x ; \omega, E)$ behaves like

$$
\varphi_{+}(x ; \omega, E)=\exp (i \alpha(\gamma(x ; \omega)-\pi)) \exp (i \sqrt{E} x \cdot \omega)(1+o(1)), \quad|x| \rightarrow \infty,
$$

where the order estimate is uniform in $x /|x| \in S^{1}$ with $|x /| x|-\omega|>\delta, \delta>0$ being fixed arbitrarily. Similarly the incoming eigenfunction $\varphi_{-}(x ; \omega, E)$ obeys

$$
\varphi_{-}(x ; \omega, E)=\exp (i \alpha(\gamma(x ;-\omega)-\pi)) \exp (i \sqrt{E} x \cdot \omega)(1+o(1))
$$

for $|x /| x|+\omega|>\delta$.

(2) If $x \in G=\left\{0<|x /| x|-\omega|<c|x|^{-1}\right\}$ for some $c>0$, then

$$
\varphi_{+}(x ; \omega, E)=(\cos \alpha \pi) \exp (i \sqrt{E} x \cdot \omega)(1+o(1)), \quad|x| \rightarrow \infty .
$$

7.3. We prove Theorem 1.2 only for the case $\omega=\hat{d}$ and $\tilde{\omega} \neq \pm \hat{d}$. A similar argument applies to the other cases. Thus we prove the asymptotic formula

$$
\begin{aligned}
f_{d}(\hat{d} \rightarrow \tilde{\omega} ; E)= & \exp \left(i \alpha_{2}(\pi-\gamma(-d ;-\tilde{\omega}))\right) f_{1}(\hat{d} \rightarrow \tilde{\omega} ; E) \\
& +\left(\cos \alpha_{1} \pi\right) \exp \left(i \alpha_{1}(\pi-\gamma(d ;-\tilde{\omega}))\right) f_{2, d}(\hat{d} \rightarrow \tilde{\omega} ; E)+o(1)
\end{aligned}
$$

when $\alpha_{2}$ is an integer. The proof is done by reduction to three lemmas. The field $b(x)=b_{1}(x)+b_{2}(x-d)$ has the flux $\alpha=\alpha_{1}+\alpha_{2}$ and it is supported in $\{x$ : $|x|<M\}$ with $M=|d|+1$. By Lemma 2.1, there exists a magnetic potential $A(x)$ associated with $b$ such that $A(x)=A_{\alpha}(x)$ for $|x|>2 M$. According to Proposition 7.1, $f_{d}(\omega \rightarrow \tilde{\omega} ; E)$ takes the form

$$
f_{d}(\omega \rightarrow \tilde{\omega} ; E)=c(E)\left(f_{\alpha}(\tilde{\omega}-\omega)-(i / 4 \pi) g_{\alpha}(\omega \rightarrow \tilde{\omega} ; E)\right), \quad \omega \neq \tilde{\omega},
$$

under natural modification of the notation in Proposition 7.1.

We study the first term $f_{\alpha}(\tilde{\omega}-\omega)$ on the right side of (7.6). Since $\alpha_{2}$ is an integer, it follows that $[\alpha]=\left[\alpha_{1}\right]+\alpha_{2}$ and $\sin \alpha \pi=(-1)^{\alpha_{2}} \sin \alpha_{1} \pi$. Hence we have 


$$
f_{\alpha}(\tilde{\omega}-\omega)=(-1)^{\alpha_{2}} \exp \left(i \alpha_{2}(\tilde{\omega}-\omega)\right) f_{\alpha_{1}}(\tilde{\omega}-\omega),
$$

where

$$
f_{\alpha_{1}}(\theta)=-\left(i \sin \alpha_{1} \pi / \pi\right) \exp \left(i\left[\alpha_{1}\right] \theta\right) F_{0}(\theta) .
$$

We further calculate

$$
\exp \left(i \alpha_{2} \tau(d ; \omega, \tilde{\omega})\right)=(-1)^{\alpha_{2}} \exp \left(i \alpha_{2}(\tilde{\omega}-\omega)\right)
$$

for $\alpha_{2}$ integer, which is independent of $d$. Thus we have

$f_{\alpha}(\tilde{\omega}-\hat{d})=\exp \left(i \alpha_{2}(\pi-\gamma(-d ;-\tilde{\omega}))\right) f_{\alpha_{1}}(\tilde{\omega}-\hat{d}), \quad f_{\alpha_{2}}(\tilde{\omega}-\omega)=0$.

We proceed to the second term

$$
g_{\alpha}(\omega \rightarrow \tilde{\omega} ; E)=\left(\psi_{+}(\omega, E), D_{M} \varphi_{-}(\tilde{\omega}, E)\right)
$$

on the right side of $(7.6)$, where $\varphi_{\mp}(\omega, E)=\varphi_{\mp}(x ; \omega, E)$, defined by $(7.3)$, is the generalized eigenfunction of $H_{\alpha}=H\left(A_{\alpha}\right)$. We change the variable $\nu=\left|m-\alpha_{1}\right|$ with $m=l-\alpha_{2}$ to obtain that

$$
\varphi_{\mp}(x ; \omega, E)=\exp \left(i \alpha_{2} \gamma(x ; \pm \omega)\right) \varphi_{\mp 1}(x ; \omega, E),
$$

where

$$
\varphi_{\mp 1}(x ; \omega, E)=\sum_{m \in Z} \exp ( \pm i \nu \pi / 2) \exp (i m \gamma(x ; \pm \omega)) J_{\nu}(\sqrt{E}|x|)
$$

with $\nu=\left|m-\alpha_{1}\right|$. It is easily seen from $(7.3)$ that $\varphi_{\mp 1}(x ; \omega, E)$ is also the eigenfunction of $H_{\alpha_{1}}=H\left(A_{\alpha_{1}}\right)$ with $A_{\alpha_{1}}(x)=\alpha_{1}\left(-x_{2} /|x|^{2}, x_{1} /|x|^{2}\right)$. For later reference, we refer to the eigenfunction $\varphi_{\mp 2}(x ; \omega, E)$ of $H_{\alpha_{2}}=H\left(A_{\alpha_{2}}\right)$. Since $\varphi_{0}(x ; \omega, E)=\exp (i \sqrt{E} x \cdot \omega)$ is expanded as

$$
\varphi_{0}(x ; \omega, E)=\sum_{l \in Z} \exp (i|l| \pi / 2) \exp (i l \gamma(x ; \omega)) J_{|l|}(\sqrt{E}|x|),
$$

$\varphi_{\mp 2}(x ; \omega, E)$ is calculated as

$$
\varphi_{\mp 2}(x ; \omega, E)=\exp \left(i \alpha_{2} \gamma(x ; \pm \omega)\right) \exp (i \sqrt{E} x \cdot \omega) .
$$

We recall that the azimuth angle $\gamma(x)$ from the positive $x_{1}$ axis satisfies (2.5). The two Hamiltonians $H_{d}=H\left(A_{1}+A_{2 d}\right)$ and $H(A)$ have the same magnetic field $b$ and hence

$$
H(A)=e^{i g} H_{d} e^{-i g}
$$


for some smooth real function $g(x)$. This function satisfies

$$
A(x)=A_{1}(x)+A_{2}(x-d)+\nabla g(x) .
$$

Since $A_{j}(x)=A_{\alpha_{j}}(x)$ for $|x|>2$, we have $\nabla g=A_{\alpha_{2}}(x)-A_{\alpha_{2}}(x-d)$ for $|x| \gg 1$ large enough. The function $g(x)$ is uniquely determined up to a constant. If $g(x) \rightarrow 0$ at infinity, then $e^{i g}$ is determined as

$$
e^{i g(x)}=\exp \left(i \alpha_{2}(\gamma(x)-\gamma(x-d))\right), \quad|x| \gg 1,
$$

by use of (2.5).

We turn back to the second term $g_{\alpha}(\omega \rightarrow \tilde{\omega} ; E)$. We rewrite this term as

$$
g_{\alpha}(\omega \rightarrow \tilde{\omega} ; E)=\left(\psi_{+d}(\omega, E), e^{-i g} D_{M} \varphi_{-}(\tilde{\omega}, E)\right),
$$

where $\psi_{+d}(\omega, E)=\psi_{+d}(x ; \omega, E)=e^{-i g(x)} \psi_{+}(x ; \omega, E)$. We define

$$
\varphi_{\mp d}(x ; \omega, E)=\exp \left(-i \alpha_{2} \gamma( \pm \omega)\right) \exp \left(i \alpha_{2} \gamma(x-d)\right) \varphi_{\mp 1}(x ; \omega, E) .
$$

Then a simple calculation using (7.9) and (7.12) yields that

$$
e^{-i g(x)} \varphi_{\mp}(x ; \omega, E)=\varphi_{\mp d}(x ; \omega, E), \quad|x| \gg 1 .
$$

Recall that $\psi_{+}(x ; \omega, E)$ satisfies $(H(A)-E) \psi_{+}=0$ and that $\psi_{+}-\varphi_{+}(x ; \omega, E)$ obeys the outgoing radiation condition at infinity. Hence $\psi_{+d}(x ; \omega, E)$ is a unique solution to $\left(H_{d}-E\right) \psi_{+d}=0$ such that $\psi_{+d}-\varphi_{+d}(x ; \omega, E)$ obeys the outgoing radiation condition. As is easily seen,

$$
H_{d}=\exp \left(i \alpha_{2} \gamma_{d}\right) H\left(A_{1}\right) \exp \left(-i \alpha_{2} \gamma_{d}\right)=\exp \left(i \alpha_{2} \gamma_{d}\right) H_{\alpha_{1}} \exp \left(-i \alpha_{2} \gamma_{d}\right)
$$

on $\Sigma_{d}=\{|x|>2\} \cap\{|x-d|>2\}$, where $\gamma_{d}(x)=\gamma(x-d)$. Hence we see that $\varphi_{+d}(x ; \omega, E)$ satisfies $\left(H_{d}-E\right) \varphi_{+d}=0$ in $\Sigma_{d}$. We now set

$$
\chi_{0}(x)=\chi(|x| / 2), \quad \chi_{0 d}(x)=\chi_{0}(x-d)
$$

and $\chi_{\infty d}(x)=1-\chi_{0}(x)-\chi_{0 d}(x)$ for the cut-off function $\chi \in C_{0}^{\infty}[0, \infty)$ with property (2.3). The function $\chi_{\infty d}$ has support in $\Sigma_{d}$ and $\chi_{0}(x) \chi_{0 d}(x)=0$ for $|d| \gg 1$. By uniqueness theorem, the solution $\psi_{+d}(x ; \omega, E)$ is represented as

$$
\psi_{+d}=\left(\chi_{\infty d}-R\left(E+i 0 ; H_{d}\right) D_{1 d}-R\left(E+i 0 ; H_{d}\right) D_{2 d}\right) \varphi_{+d},
$$

where

$$
D_{1 d}=\left[\chi_{0}, H_{d}\right], \quad D_{2 d}=\left[\chi_{0 d}, H_{d}\right] .
$$


We consider the term $e^{-i g} D_{M} \varphi_{-}(x ; \tilde{\omega}, E)$ on the right side of $(7.13)$, where $D_{M}=\left[H(A), \chi_{\infty M}\right]$ is defined by (7.5) with $M=|d|+1$. Since

$$
\chi_{\infty M}(H(A)-E) \varphi_{-}=\chi_{\infty M}\left(H_{\alpha}-E\right) \varphi_{-}=0,
$$

we may write

$$
e^{-i g} D_{M} \varphi_{-}=e^{-i g}(H(A)-E) \chi_{\infty M} \varphi_{-}=\left(H_{d}-E\right) \chi_{\infty M} e^{-i g} \varphi_{-} .
$$

On the other hand, $\varphi_{-d}(x ; \tilde{\omega}, E)$ also satisfies $\left(H_{d}-E\right) \varphi_{-d}=0$ in $\Sigma_{d}$, and hence $\chi_{\infty d}\left(H_{d}-E\right) \varphi_{-d}=0$. Thus $e^{-i g} D_{M} \varphi_{-}(x ; \tilde{\omega}, E)$ is represented as

$$
e^{-i g} D_{M} \varphi_{-}=\left(H_{d}-E\right)\left(\chi_{\infty M} e^{-i g} \varphi_{-}-\chi_{\infty d} \varphi_{-d}\right)+\left(D_{1 d}+D_{2 d}\right) \varphi_{-d},
$$

where $D_{1 d}$ and $D_{2 d}$ are defined in (7.18). By (7.15), $e^{-i g} \varphi_{-}-\varphi_{-d}$ has compact support and $\psi_{+d}$ obeys $\left(H_{d}-E\right) \psi_{+d}=0$. We combine (7.19) with (7.17) to obtain that $g_{\alpha}(\omega \rightarrow \tilde{\omega} ; E)$ admits the decomposition

$$
g_{\alpha}(\omega \rightarrow \tilde{\omega} ; E)=s_{1}(\omega \rightarrow \tilde{\omega} ; d)+s_{2}(\omega \rightarrow \tilde{\omega} ; d)+s_{12}(d)+s_{21}(d),
$$

where

$$
\begin{aligned}
s_{1}(\omega \rightarrow \tilde{\omega} ; d) & =\left(\left(\left(1-\chi_{0}\right)-R\left(E+i 0 ; H_{d}\right) D_{1 d}\right) \varphi_{+d}(\omega, E), D_{1 d} \varphi_{-d}(\tilde{\omega}, E)\right), \\
s_{2}(\omega \rightarrow \tilde{\omega} ; d) & =\left(\left(\left(1-\chi_{0 d}\right)-R\left(E+i 0 ; H_{d}\right) D_{2 d}\right) \varphi_{+d}(\omega, E), D_{2 d} \varphi_{-d}(\tilde{\omega}, E)\right), \\
s_{j k}(d) & =-\left(R\left(E+i 0 ; H_{d}\right) D_{j d} \varphi_{+d}(\omega, E), D_{k d} \varphi_{-d}(\tilde{\omega}, E)\right)
\end{aligned}
$$

with $\varphi_{\mp d}(\omega, E)=\varphi_{\mp d}(x ; \omega, E)$.

\section{Lemma 7.1.}

$$
s_{12}(d), \quad s_{21}(d) \longrightarrow 0
$$

Lemma 7.2. Let $\chi_{0}(x)$ be as in (7.16) and let $D_{1}=\left[\chi_{0}, H\left(A_{1}\right)\right]$. Then $s_{1}(d)=s_{1}(\hat{d} \rightarrow \tilde{\omega} ; d)$ behaves like

$$
s_{1}(d)=\exp \left(i \alpha_{2}(\pi-\gamma(-d ;-\tilde{\omega}))\right) g_{\alpha_{1}}(\hat{d} \rightarrow \tilde{\omega} ; E)+o(1)
$$

where

$$
g_{\alpha_{1}}(\omega \rightarrow \tilde{\omega} ; E)=\left(\psi_{+1}(\omega, E), D_{1} \varphi_{-1}(\tilde{\omega}, E)\right)
$$

and $\psi_{+1}(\omega, E)=\psi_{+1}(x ; \omega, E)$ is a unique solution to $\left(H\left(A_{1}\right)-E\right) \psi_{+1}=0$ such that $\psi_{+1}-\varphi_{+1}(x ; \omega, E)$ obeys the outgoing radiation condition at infinity. 
Lemma 7.3. Let $D_{2}=\left[\chi_{0}, H\left(A_{2}\right)\right]$. Then $s_{2}(d)=s_{2}(\hat{d} \rightarrow \tilde{\omega} ; d)$ with $\tilde{\omega} \neq-\hat{d}$ behaves like

$$
\begin{aligned}
s_{2}(d)= & \left(\cos \alpha_{1} \pi\right) \exp \left(i \alpha_{1}(\pi-\gamma(d ;-\tilde{\omega}))\right) \\
& \times \exp (-i \sqrt{E} d \cdot(\tilde{\omega}-\hat{d})) g_{\alpha_{2}}(\hat{d} \rightarrow \tilde{\omega} ; E)+o(1),
\end{aligned}
$$

where

$$
g_{\alpha_{2}}(\omega \rightarrow \tilde{\omega} ; E)=\left(\psi_{+2}(\omega, E), D_{2} \varphi_{-2}(\tilde{\omega}, E)\right)
$$

and $\psi_{+2}(x ; \omega, E)$ is a unique solution to $\left(H\left(A_{2}\right)-E\right) \psi_{+2}=0$ such that $\psi_{+2}-$ $\varphi_{+2}(x ; \omega, E)$ obeys the outgoing radiation condition at infinity.

If we recall the representation for the amplitude in Proposition 7.1, then the desired asymptotic formula for $f_{d}(\hat{d} \rightarrow \tilde{\omega} ; E)$ is obtained from $(7.8)$ as an immediate consequence of the three lemmas above.

7.4. We shall complete the proof of the theorem by proving the three lemmas above. The proof of the lemmas is based on Lemma 3.2.

Proof of Lemma 7.1. Recall that $\varphi_{\mp d}(x ; \omega, E)$ is defined by (7.14). We apply Proposition 7.2 to the eigenfunction $\varphi_{\mp 1}(x ; \omega, E)$ of $H_{\alpha_{1}}$. Then

$$
\int_{|x-d|<4}\left|\varphi_{\mp d}(x ; \omega, E)\right|^{2} d x=O(1)
$$

is bounded uniformly in $d$, and hence

$$
\int_{|x-d|<4}\left|\nabla \varphi_{\mp d}(x ; \omega, E)\right|^{2} d x=O(1)
$$

is also uniformly bounded by elliptic estimate. Let $g_{1}$ and $g_{2}$ be bounded functions with support in $\{|x|<4\}$ and $\{|x-d|<4\}$ respectively. Then we have

$$
\left\|g_{1} R\left(E+i 0 ; H_{d}\right) g_{2}\right\|=o(1)
$$

by Lemma 3.2. This, together with (7.20), completes the proof.

Proof of Lemma 7.2. Let $\chi_{0}(x)=\chi(|x| / 2)$ be as in (7.16). Recall that $D_{1 d}=\left[\chi_{0}, H_{d}\right]$ is defined by (7.18). The coefficients of $D_{1 d}$ have support in $Q_{1}=\{2<|x|<4\}$. Hence it follows from Lemma 3.2 that

$$
s_{1}(d)=\left(\left(\left(1-\chi_{0}\right)-R\left(E+i 0 ; H\left(A_{1}\right)\right) D_{1 d}\right) \varphi_{+d}(\hat{d}, E), D_{1 d} \varphi_{-d}(\tilde{\omega}, E)\right)+o(1) .
$$


The operator $H_{d}$ coincides with $K_{1 d}=H\left(A_{1}+\nabla \eta_{2 d}\right)=p_{2 d} H\left(A_{1}\right) q_{2 d}$ on $Q_{1}$, so that

$$
D_{1 d}=p_{2 d}\left[\chi_{0}, H\left(A_{1}\right)\right] q_{2 d}=p_{2 d} D_{1} q_{2 d} .
$$

By (4.8), $p_{2 d}(x)$ and $q_{2 d}(x)=1 / p_{2 d}(x)$ behave like

$$
p_{2 d}(x)=e^{i \alpha_{2} \pi}+O\left(|d|^{-1}\right), \quad q_{2 d}(x)=e^{-i \alpha_{2} \pi}+O\left(|d|^{-1}\right)
$$

on $Q_{1}$. Thus we have

$$
s_{1}(d)=\left(\left(\left(1-\chi_{0}\right)-R\left(E+i 0 ; H\left(A_{1}\right)\right) D_{1}\right) \varphi_{+d}(\hat{d}, E), D_{1} \varphi_{-d}(\tilde{\omega}, E)\right)+o(1) .
$$

By $(7.14), \varphi_{\mp d}(x ; \omega, E)$ behaves like

$$
\varphi_{\mp d}(x ; \omega, E)=\exp \left(i \alpha_{2}(\gamma(-d)-\gamma( \pm \omega))\right) \varphi_{\mp 1}(x ; \omega, E)+O\left(|d|^{-1}\right)
$$

uniformly on $Q_{1}$, and

$$
\psi_{+}(x ; \omega, E)=\left(\left(1-\chi_{0}\right)-R\left(E+i 0 ; H\left(A_{1}\right)\right) D_{1}\right) \varphi_{+1}(x ; \omega, E)
$$

is a unique solution to $\left(H\left(A_{1}\right)-E\right) \psi_{+}=0$ such that $\psi_{+}-\varphi_{+1}$ satisfies the outgoing radiation condition. Hence we have

$$
s_{1}(d)=\exp \left(i \alpha_{2}(\gamma(\tilde{\omega})-\gamma(-\hat{d}))\right) g_{\alpha_{1}}(\hat{d} \rightarrow \tilde{\omega} ; E)+o(1) .
$$

The phase factor on the right side equals $\exp \left(i \alpha_{2}(\pi-\gamma(-d ;-\tilde{\omega}))\right)$ for $\alpha_{2}$ integer. This completes the proof.

Proof of Lemma 7.3. We repeat the same argument as in the proof of Lemma 7.2. Let $D_{2 d}=\left[\chi_{0 d}, H_{d}\right]$ be as in (7.18). The coefficients of $D_{2 d}$ have support in $Q_{2 d}=\{2<|x-d|<4\}$. Set $D_{d}=\left[\chi_{0 d}, H\left(A_{2 d}\right)\right]$. Then $D_{2 d}$ is calculated as

$$
D_{2 d}=\left[\chi_{0 d}, K_{2 d}\right]=p_{1 d}\left[\chi_{0 d}, H\left(A_{2 d}\right)\right] q_{1 d}=p_{1 d} D_{d} q_{1 d}
$$

on $Q_{2 d}$, and

$$
p_{1 d}(x)=e^{i \alpha_{1} \pi}+O\left(|d|^{-1}\right), \quad q_{1 d}(x)=e^{-i \alpha_{1} \pi}+O\left(|d|^{-1}\right)
$$

on $Q_{2 d}$ by (4.7). Hence we have

$$
s_{2}(d)=\left(\left(\left(1-\chi_{0 d}\right)-R\left(E+i 0 ; H\left(A_{2 d}\right)\right) D_{d}\right) \varphi_{+d}(\hat{d}, E), D_{d} \varphi_{-d}(\tilde{\omega}, E)\right)+o(1)
$$


by Lemma 3.2. We analyse the behavior of $\varphi_{+d}(\hat{d}, E)$ and $\varphi_{-d}(\tilde{\omega}, E)$ over $Q_{2 d}$. Since

$$
\exp \left(-i \alpha_{2} \gamma( \pm \omega)\right) \exp \left(i \alpha_{2} \gamma(x-d)\right)=\exp \left(i \alpha_{2} \gamma(x-d ; \pm \omega)\right)
$$

it follows from (7.14) that

$$
\varphi_{\mp d}(x ; \omega, E)=\exp \left(i \alpha_{2} \gamma(x-d ; \pm \omega)\right) \varphi_{\mp 1}(x ; \omega, E) .
$$

We apply Proposition $7.2(2)$ to $\varphi_{+1}(x ; \hat{d}, E)$ to obtain that

$$
\varphi_{+d}(x ; \hat{d}, E)=\left(\cos \alpha_{1} \pi\right) \exp \left(i \alpha_{2} \gamma(x-d ;-\hat{d})\right) \varphi_{0}(x ; \hat{d}, E)+o(1)
$$

on $Q_{2 d}$, where $\varphi_{0}(x ; \omega, E)=\exp (i \sqrt{E} x \cdot \omega)$. Hence we see from $(7.11)$ that

$$
\varphi_{+d}(x ; \hat{d}, E)=\left(\cos \alpha_{1} \pi\right) \exp (i \sqrt{E} d \cdot \hat{d}) \varphi_{+2}(x-d ; \hat{d}, E)+o(1)
$$

on $Q_{2 d}$. On the other hand, Proposition $7.2(1)$ applied to $\left.\varphi_{-1}(x ; \tilde{\omega}, E)\right)$ with $\tilde{\omega} \neq-\hat{d}$ yields

$$
\varphi_{-d}(x ; \tilde{\omega}, E)=\exp \left(i \alpha_{1}(\gamma(d ;-\tilde{\omega})-\pi)\right) \exp (i \sqrt{E} d \cdot \tilde{\omega}) \varphi_{-2}(x-d ; \tilde{\omega}, E)+o(1)
$$

on $Q_{2 d}$. These two relations complete the proof.

\section{References}

[1] Afanasiev, G. N., Topological Effects in Quantum Mechanics, Kluwer Academic Publishers, 1999.

[2] Aharonov, Y. and Bohm, D., Significance of electromagnetic potential in the quantum theory, Phys. Rev., 115 (1959), 485-491.

[3] Berry, M. V., Chambers, R. G., Large, M. D., Upstill, C. and Walmsley, J. C., Wavefront dislocations in the Aharonov-Bohm effect and its water wave analogue, Eur. J. Phys., 1 (1980), 154-162.

[4] Ikebe, T. and Saitō, Y., Limiting absorption method and absolute continuity for the Schrödinger operators, J. Math. Kyoto Univ., 7 (1972), 513-542.

[5] Isozaki, H. and Kitada, H., A remark on the micro-local resolvent estimates for two body Schrödinger operators, Publ. RIMS, Kyoto Univ., 21 (1985), 889-910.

[6] - Scattering matrices for two-body Schrödinger operators, Sci. Papers Coll. of Arts and Sci., Univ. Tokyo, 35 (1985), 81-107.

[7] Ito, H. T. and Tamura, H., Aharonov-Bohm effect in scattering by point-like magnetic fields at large separation, Ann. H. Poincaré, 2 (2001), 309-359.

[8] Kostrykin, V. and Schrader, R., Cluster properties of one particle Schrödinger operators. II, Rev. Math. Phys., 10 (1998), 627-683.

[9] Loss, M. and Thaller, B., Scattering of particles by long-range magnetic fields, Ann. of Phys., 176 (1987), 159-180.

[10] Ohnuki, Y., Aharonov-Bohm kōka (in Japanese), Butsurigaku saizensen 9, Kyōritsu syuppan, 1984. 
[11] Reed, M. and Simon, B., Methods of Modern Mathematical Analysis, Vol II, Academic Press, 1976.

[12] Ruijsenaars, S. N. M., The Aharonov-Bohm effect and scattering theory, Ann. of Phys., 146 (1983), 1-34.

[13] Tamura, H., Shadow scattering by magnetic fields in two dimensions, Ann. Inst. Henri Poincaré, 63 (1995), 253-276.

[14] - Magnetic scattering at low energy in two dimensions, Nagoya Math. J., 155 (1999), 95-151.

[15] Yafaev, D., Scattering Theory: Some old and new problems, Lec. Notes in Math., 1735 (2000), Springer. 